\title{
Highlights
}

\section{Ontology Integration: Approaches and Challenging Issues}

Inès Osman, Sadok Ben Yahia, Gayo Diallo

- Eliciting key definitions and notions related to the ontology integration area.

- Reporting and analyzing integration principles, consequences and repair techniques.

- Briefly reviewing the most relevant research works on the ontology integration area.

- Performing a holistic ontology integration to highlight open issues from the survey.

- Improving the ontology matching will considerably improve the ontology integration. 


\title{
Ontology Integration: Approaches and Challenging Issues
}

\author{
Inès Osman ${ }^{\mathrm{a}, *}$, Sadok Ben YAHIA ${ }^{\mathrm{b}}$, Gayo Diallo ${ }^{\mathrm{c}}$ \\ ${ }^{a}$ LIPAH - LR11ES14, Faculty of Sciences of Tunis, University of Tunis El Manar, 2092 Tunis, Tunisia \\ ${ }^{b}$ Department of Software Science, Tallinn University of Technology, Estonia \\ ${ }^{c}$ INRIA SISTM, Team ERIAS - INSERM Bordeaux Population Health Research Center, University of Bordeaux, F-33000 Bordeaux, France
}

\begin{abstract}
In recent years, the decentralized development of ontologies has led to the generation of multiple ontologies of overlapping knowledge. This heterogeneity problem can be tackled by integrating existing ontologies to build a single coherent one. Ontology integration has been investigated during the last two decades, but it is still a challenging task. In this article, we provide a comprehensive survey of all ontology integration aspects. We discuss related notions and scrutinize existing techniques and literature approaches. We also detail the role of ontology matching in the ontology integration process. Indeed, the ontology community has adopted the splitting of the ontology integration problem into matching, merging and repairing sub-tasks, where matching is a necessary preceding step for merging, and repairing can be included in the matching process or performed separately. Ontology matching and merging systems have become quite proficient, however the trickiest part lies in the repairing step. We also focus on the case of a holistic integration of multiple heterogeneous ontologies, which needs further exploration. Finally, we investigate challenges, open issues, and future directions of the ontology integration and matching areas.
\end{abstract}

Keywords: Ontology Integration, Ontology Merging, Ontology Matching, Alignment Repair, Coherence Principle, Conservativity Principle

2010 MSC: 00-01, 99-00

\section{Introduction}

Ontologies have been shown to be the best means for communicating and sharing knowledge between people and machines. They provide a common understanding of a given domain thanks to their shared vocabulary (i.e., terms with unified meanings). They can be used ( $i$ ) as a query model for data sources, or (ii) as a basis for the integration of heterogeneous resources, e.g., Web pages, XML documents, and relational databases, etc [1, 2].

However, ontologies do not completely solve the data integration problem. Indeed, one cannot expect all organizations to agree on the use of a common ontology. Therefore, it is unlikely that a global ontology covering all distributed systems could be developed. For instance, different applications, that have annotated their data with syntactically different but similar ontologies, work independently and cannot communicate, inter-operate or interact with each other.

10 In fact, ontologies may cover the same domain or similar domains with different delimitations, perspectives/viewpoints, conceptual designs, granularity (levels of detail), and naming conventions. Overall, several competing, incomplete and overlapping ontologies could cover a given domain; so heterogeneity issues are inevitable. These issues arise because ontology building can never be a deterministic process in which different ontology developers make similar designing decisions. In practice, ontologies are developed independently of each other, in multiple places, by different 15 communities and designers, for different applications, and with different requirements, prerequisites, and tools.

To leverage their power, ontologies need to overcome this semantic heterogeneity issue by integrating their distributed knowledge [3]. An ontology integration process generates a coherent integrated ontology from multiple

\footnotetext{
${ }^{*}$ Corresponding author

Email addresses: ines.osman@fst.utm.tn (Inès Osman), sadok.ben@taltech.ee (Sadok BEN YAHIA), gayo.diallo@u-bordeaux.fr (Gayo Diallo)
} 
input ontologies. It is an ontology reuse process [4-6] where ontologies can be reused globally or partially. Ontology integration in Big Data is impossible to achieve due to an extensive amount of data. However, integrating ontologies is strongly considered in ontology development tasks (i.e. when building a new ontology), since building ontologies from scratch is a labor-intensive and costly task. For example, ontology integration is useful for building application-specific ontologies (e.g., ontologies of chatbot applications), or for building generic domain ontologies that can be extended or customized for specific applications. Here are some examples where it is necessary to integrate ontologies [7]:

1. Private companies may be interested in using community standard ontologies together with company-specific ontologies;

2. Collaborative companies may not only share physical assets, but may also share knowledge that needs to be integrated;

3. Companies may need to update their current ontology by adding new knowledge [8] because of new business processes requirements;

4. Applications that rely on domain ontologies may need to use ontologies covering different perspectives on one domain;

5. Ontology developers may be interested in reusing already existing ontologies as the basis for the development of new ontologies for broader domains [9]. They extend or combine them with other sub-domain ontologies.

It is worth noting that (database) schema integration and ontology integration areas have much in common [10].

Their main approaches usually comprise two primary steps: $(i)$ first, the matching step which reconciles the differences by identifying semantic correspondences (mainly similarities) between different elements, using a similarity computation; then (ii) the merging step, which exploits the result of the matching step by merging or linking the matched elements, to produce a new unified view. An optional repairing step can be either included during the matching step, or be a standalone third step. Therefore, ontology matching is a necessary internal phase for ontology integration; and the 40 improvement of ontology matching results will considerably improve ontology integration results. Automated ontology matching systems have become very efficient in the discovery of simple equivalence correspondences between named entities, especially between concepts. Despite this progress, there are still many issues and challenges to face in the ontology integration area. We have reported many surveys on ontology matching and its different existing approaches in the literature, e.g., [11-19], etc. However, there is a huge lack of literature surveys dedicated to ontology integration.

In this paper, we are interested in reviewing the topic of ontology integration, and some associated aspects belonging to the ontology matching area. More precisely, the paper makes a thorough literature review regarding the different notions, approaches, issues and future avenues of the ontology integration area, with a minor emphasis on the ontology matching sub-area which plays an important role in the ontology integration process.

The remainder of the paper is structured as follows. Section 2 recalls some background knowledge about ontology, 50 including ontology definitions and the $O W L$ Description Logics-based language. Section 3 describes frequently used notions that are closely related to the topic of ontology integration, such as ontology matching, alignment, mapping, correspondence, and relation. After defining the key notions of ontology integration and ontology merging encountered in the literature, section 4 describes their different existing techniques. Section 5 explains the principles of ontology integration, the reasons behind the emergence of some issues in an integrated ontology, and the different strategies to their resolution. Section 6 briefly reviews the related work on ontology integration and merging, and sheds light on some initial observations. Section 7 gathers all the evaluation metrics used in the state of the art. Section 8 introduces two ontology integration algorithms, uses them to perform a holistic integration of multiple ontologies, then discusses the results and highlights the corresponding difficulties and challenges. Section 9 derives some interesting findings and sketches future directions from this survey. Finally, section 10 concludes the paper with a short summary.

\section{Background: Ontology \& OWL}

According to Studer et al. [20], "an ontology is a formal, explicit specification of a shared conceptualization (of a domain of discourse)". It is a set of triplets < $<$ entity $y_{1}$, relation, entity $>$ (a.k.a. < subject, predicate, object $>$ ). It can be viewed as a labelled directed graph whose nodes are entities, and edges are relations. Nodes are labelled by entity names, and edges are labelled by relation names. There are seven types of entities in an ontology: concepts (sets of individuals, or semantic categories), object properties (relationships or associations), datatype properties (or attributes), 
annotation properties, individuals (instances, or objects), datatypes, and data values (or data literals). Entities are the union of all the latter ones [17]. However, in general, the are four main ontology entities, which are concepts, object properties, datatype properties, and individuals. Entities can be named entities or anonymous entities. Named entities are entities that are clearly identifiable by name, e.g., a concept "Person". However, anonymous entities are not identifiable since they do not have a name. They are generally complex expressions, such as class or property restrictions, used in the description of named entities. For example, an anonymous class $A$ can be the union/intersection of three particular named classes; To assert that a given named class $B$ is disjoint to the union/intersection of the three named classes, it should be declared that the named class $B$ is disjoint to the anonymous class $A$.

In RDF-based languages such as RDF (Resource Description Framework) [21, 22], RDFS (RDF Schema) [23] and OWL (Web Ontology Language) [24, 25], a concept is called a "class" or a "class of individuals". A concept can instantiate zero or more individuals; therefore, individuals are called the members of the concept or the extension of the concept. An individual is instantiated by a class using the built-in "type" relation. This instantiation is called a class assertion. These individuals can be linked through the object and datatype properties that are defined in the ontology. The domain of a property is its subject, and the range of a property is its object (domain $\leftarrow$ property $\rightarrow$ so range). An object property links an instance of a given class/domain to an instance of a given class/range. This is called an object property assertion. While, a datatype property links an instance of a given class/domain to a data value of a given datatype/range, such as string, integer, real, boolean, etc. This is called a datatype property assertion. Finally, annotation properties are used to annotate the whole ontology or entities of the ontology with a human-readable description, such as comments, entity labels, and ontology version information, etc.

Concepts and properties are generally organized within hierarchies since they are linked by the built-in subsumption relation. The latter is a taxonomic, "is-a", parent/child, hypernymy/hyponymy, super-entity/sub-entity, super-type/subtype, generalization/specialization, or broader/narrower relation. It can also be called a partial order, inheritance, or general concept inclusion (GCI) relation. Thus, three hierarchies can exist in an ontology: a class hierarchy, an object property hierarchy, and a datatype property hierarchy. The class hierarchy can be viewed as a tree-based model [26].

90 The latter forms a rooted acyclic graph structure [27] due to the subsumption relation that makes each concept subsumed by a single direct super-concept/parent (except the root concept owl:Thing). Nevertheless, in the case of multiple inheritance, an ontology becomes a network-based model that can contain multiple $i s$ - $a$ paths leading to the same concept [26]. There are many other built-in relations that can link classes, properties or instances, such as equivalence and disjointness relations, etc (See Subsection 3.5 ).

In the abstract syntax, an ontology is a set of logical and non-logical axioms (i.e., rules or constraints) that describe and express the entities of a domain of discourse and their associated declarations and assertions. Conceptual elements (i.e., classes and properties) and the axioms that restrict their interpretation are called the ontology schema, structure, intensional level, or T-Box (for Terminological Box). Whereas, individuals and their axioms (facts), that instantiate the T-Box information, are called the ontology data, instance data, extensional level, or A-Box (for Assertional Box).

An IRI (Internationalized Resource Identifier) is considered as a unique name that identifies an ontology or an entity. The full IRI, a.k.a. the prefixed name, of an entity is composed of a prefix and a suffix separated by the symbol "\#" (or by the symbol "/" or ":"). The entity prefix is usually the IRI of the ontology in which the entity appears (e.g., the IRI of the current ontology, or the IRI of another existing ontology). The entity suffix is the local, abbreviated, or short name of the entity.

$$
\begin{gathered}
\text { Entity IRI/Name = IRI Prefix + "\#" + IRI Suffix } \\
\text { Entity IRI/Name = Ontology IRI + "\#" + Entity Short Name }
\end{gathered}
$$

The Web Ontology Language (OWL) [28] is an XML-based language for modeling and expressing ontologies. It is the most widely used knowledge representation language in the framework of the Semantic Web [29, 30]. OWL became a World Wide Web Consortium (W3C) ${ }^{1}$ recommendation in 2004; and OWL 2 [25], which is a richer version of OWL, became a W3C recommendation in 2009. Compared to other XML-based languages such as RDF and RDFS, 10 OWL endows machines with a greater ability to interpret Web content thanks to its rich vocabulary and underlying formal semantics of Description Logics. Description Logics (DL) [31] are logics that are specifically designed to represent and reason on structured knowledge. They can be considered as decidable fragments of First-Order logic (FOL). Therefore, ontologies are actually logical theories.

\footnotetext{
https://www.w3.org
} 


\section{Key Notions Related to Ontology Matching} dence, relation, etc., which are very ambiguous and sometimes misused. Therefore, it is of utmost importance to provide a consensual definition for each term. In the following, we will refer to these terms as defined in the remainder of this section.

\subsection{Ontology Matching}

In general, ontology matching - also called ontology alignment although it is not preferred-is the process of discovering semantic correspondences between entities coming from different ontologies, and then generating an alignment. This process takes as input a set of $N$ ontologies to match, denoted by $\Omega$, and returns a set of correspondences which constitutes an alignment $\mathcal{A}$. According to Euzenat and Shvaiko [17], the ontology matching process can be seen as a function $f(\Omega, \mathcal{A}, p, r)=\mathcal{A}^{\prime}$ which, from a set of ontologies $\Omega$, an input alignment $\mathcal{A}$, a set of parameters $p$ and a set of resources $r$, returns an alignment $\mathcal{A}^{\prime}$ between entities of the input ontologies. An algorithm that performs an ontology matching process is called an ontology matcher.

In the ontology matching area, the matched entities are usually classes and properties, which is precisely referred to as schema matching or T-Box matching. However, when it comes to matching individuals, it is rather about instance matching, object matching, coreference resolution or link discovery. Ontology matching can either refer to matching an T-Box [32]. We should note that most of the ontology matching solutions typically rely much more on schema-level information than on instance-level information or both schema- and level- information [17].

In the following, we will categorize different ontology matching types, and describe each one separately.

\subsubsection{Simple vs. Complex Ontology Matching}

The complexity/expressiveness of the resulting correspondences generates two types of ontology matching: the simple ontology matching for generating simple correspondences; and the complex ontology matching for generating complex correspondences.

- Simple Ontology Matching is restricted to matching named entities, which are entities identified by IRIs/namespaces, between ontologies. It is also called one-to-one ontology matching because, in case of matching two ontologies, it matches one named entity from the source ontology to one named entity from the target ontology $(1: 1)$. An exhaustive matching approach would compare every named entity in the first ontology to every named entity in the second ontology [32].

- Complex Ontology Matching extends the simple ontology matching by also matching anonymous entities, which are entities in a term building expression or formulas. That is, it can find correspondences between complex expressions. The latter are new entities constructed by named entities using either logical constructors/connectors of a logical language (DL or FOL), e.g. property restrictions, or using transformation functions of datatype values such as operations on strings (e.g., a string concatenation), or using arithmetic operations such as a conversion [33, 34]. The complex matching is also referred to as one-to-many, many-to-one, or many-to-many ontology matching. Indeed, in case of matching two ontologies - which is the most common case,

- it can match one named entity from the first ontology to an expression (i.e., a construction of named entities, or a logical formulation of named entities) from the second ontology $(1: n)$;

- it can match an expression from the first ontology to one named entity from the second ontology $(m: 1)$; or

- it can match an expression from the first ontology to an expression from the second ontology $(m: n)$.

An exhaustive matching approach would at least compare every named entity in one ontology to all possible 


\subsubsection{Pairwise vs. Holistic Ontology Matching}

The number of input ontologies to be matched generates two types of ontology matching: the pairwise or binary ontology matching [35] for matching two input ontologies; and the holistic or N-ary ontology matching, a.k.a. the multiple ontology matching [17], for matching several input ontologies.

- Pairwise Ontology Matching matches a source ontology to a target ontology. It takes as input a pair of ontologies to match $\Omega=\left\{O_{1}, O_{2}\right\}$ and returns an alignment $\mathcal{A}$ between these two ontologies. A matching approach needs to handle the search space between named and/or anonymous entities of only two ontologies [32].

- Holistic Ontology Matching generalizes the pairwise ontology matching by simultaneously inspecting multiple ontologies within the matching process. It takes as input a set of ontologies $\Omega=\left\{O_{1}, O_{2}, \ldots, O_{N}\right\}$ with $N>2$, processes all input ontologies at once (in one run) and returns an alignment $\mathcal{A}$ between these ontologies [36]. A matching approach needs to handle a much larger search space between named and/or anonymous entities of $N$ ontologies instead of two [32]. To facilitate this task, a holistic matching can rely on a set of pairwise alignments that are obtained by a pairwise matching. State-of-the-art works have implemented the two following more simple approaches:

- N-way Ontology Matching [35] also takes as input a set of ontologies $\Omega=\left\{O_{1}, O_{2}, \ldots, O_{N}\right\}$ with $N>2$, and returns an alignment $\mathcal{A}$ between all these ontologies. However, it implements a series of 2-way/pairwise ontology matching, by incrementally aggregating the two current matched ontologies.

- ( $\left.\begin{array}{c}\mathbf{N} \\ 2\end{array}\right)$-way Ontology Matching also takes as input a set of ontologies $\Omega=\left\{O_{1}, O_{2}, \ldots, O_{N}\right\}$ with $N>2$, and returns an alignment $\mathcal{A}$. However, it implements a series of pairwise matching on all the available pairs of ontologies and aggregates all their resulting alignments. For example, if $\Omega=\left\{O_{1}, O_{2}, O_{3}\right\}$, then the alignment $\mathcal{A}$ can be defined as $\mathcal{A}=\mathcal{A}_{12} \cup \mathcal{A}_{13} \cup \mathcal{A}_{23}$. This approach will separately make $\left(\begin{array}{l}N \\ 2\end{array}\right)$ pairwise ontology matching runs [37], which corresponds to the complete pairwise alignment space between all input ontologies.

\subsubsection{Oriented vs. Non-Oriented Ontology Matching}

In the pairwise ontology matching case, the direction, in which the two input ontologies are matched, generates two types of ontology matching: the oriented ontology matching for performing a unidirectional matching from a source ontology to a target ontology; and the non-oriented ontology matching, which is the most common, for performing a bidirectional matching, applicable in both directions, i.e. from one ontology to the other, and vice versa [2, 11].

- Oriented Ontology Matching takes as input two ontologies and returns an oriented alignment containing "one-way" correspondences all oriented in the same direction.

- Non-Oriented Ontology Matching takes as input two ontologies and returns a non-oriented alignment containing "two-way" correspondences oriented in both directions.

\subsubsection{Compound vs. Ternary Ontology Matching}

When it is about a holistic complex matching, the origin of the entities that are composing a complex expression of a given correspondence generates two types of ontology matching: the compound ontology matching for matching expressions that involve a combination of entities originating from two or more input ontologies; and the ternary compound ontology matching for matching a source ontology to two target ontologies [38, 39], which is a special case of a compound matching.

- Compound Ontology Matching [38] takes as input at least three ontologies to match $\Omega=\left\{O_{1}, O_{2}, \ldots, O_{N}\right\}$ with $N>2$, and returns a compound alignment $\mathcal{A}$. It can discover correspondences involving complex expressions that are constructed by existing named entities coming from two or more input ontologies. It needs to handle a large search space between entities of $N$ ontologies, and also be able to compose expressions using entities from different ontologies. An exhaustive matching approach would at least compare every named entity in one ontology to all possible combinations of named entities in the other $(N-1)$ ontologies [32]. 
- Ternary Ontology Matching [38] takes as input three ontologies to match $\Omega=\left\{O_{1}, O_{2}, O_{3}\right\}$, and returns a ternary compound alignment $\mathcal{A}$ containing equivalence correspondences. It matches named entities from the first input ontology to expressions involving named entities from the second and third input ontologies. It needs to handle a search space between entities of three ontologies, and also be able to compose expressions using entities from two different ontologies. An exhaustive matching approach would compare every named entity in the first ontology to all possible combinations of named entities in the other two ontologies [32]. This is the simplest case of a compound matching.

In the literature, a simple pairwise non-oriented matching is called, in short, an ontology matching. In the complexity scale, we categorize ontology matching levels in an ascending order as follows: simple, complex, ternary, and finally compound matching.

\subsection{Ontology Alignment}

A semantic alignment is a set of semantic correspondences between two or more matched ontologies, denoted by $\mathcal{A}=\left\{c_{1}, c_{2}, \ldots, c_{n}\right\}$, and stored separately. It is the result of the ontology matching process. Alignments can be used in various tasks, such as query response or distributed querying, query rewriting, logical reasoning on ontologies, data interlinking or Semantic Web browsing, ontology transformation, and ontology integration \& merging. Following the aforementioned ontology matching types, we distinguish eight alignment types:

- Simple Alignment only contains simple entity-to-entity correspondences. The most used format for representing simple pairwise alignments is the RDF Alignment format [40], a.k.a. the Alignment format or the Alignment API format - where Alignment is written with a capitalized "A". This format is expressed in RDF. It is the most consensual ontology alignment format, despite its simplicity and its lack of expressiveness and thus, inability to represent all kinds of correspondences.

- Complex Alignment contains at least one complex correspondence-expressed as a logical rule. In this case, $E D O A L^{3}$ (Expressive and Declarative Ontology Alignment Language) [40] is the most used format for representing complex pairwise alignments. It extends the Alignment format by also allowing to express complex correspondences.

- Pairwise Alignment, a.k.a. binary alignment [35], only involves two ontologies to match: a source ontology and a target ontology; and only contains correspondences linking two entities, each coming from an ontology.

- Holistic Alignment, a.k.a. multi-alignment or multialignment [17], involves more than two ontologies and contains at least one correspondence that links different entities coming from more than two input ontologies.

- Oriented Alignment, a.k.a. mapping [17] or directional alignment [41], is the directed or oriented version of a pairwise alignment [17], denoted $\mathcal{A}_{O_{1} \rightarrow O_{2}}$ where the source ontology is $O_{1}$ and the target ontology is $O_{2}$. Correspondences are total functions rather than relations [2]. When an alignment is unidirectional, $\mathcal{A}_{\mathrm{O}_{1} \rightarrow \mathrm{O}_{2}}$ is not the inverse of $\mathcal{A}_{\mathrm{O}_{2} \rightarrow O_{1}}$ [41].

- Non-Oriented Alignment is a non-directed or non-oriented pairwise alignment, denoted $\mathcal{A}$ or $\mathcal{A}_{O_{1} \leftrightarrow O_{2}}$ and briefly called an alignment. When an alignment is a bidirectional one, $\mathcal{A}_{\mathrm{O}_{1} \leftrightarrow \mathrm{O}_{2}}$ is the inverse of $\mathcal{A}_{\mathrm{O}_{2} \leftrightarrow O_{1}}$. Since a binary relation can be decomposed into a pair of total functions, the bidirectional alignment can be considered as a pair of oriented alignments, so $\mathcal{A}_{O_{1} \leftrightarrow O_{2}}=\mathcal{A}_{O_{1} \rightarrow O_{2}}+\mathcal{A}_{O_{2} \rightarrow O_{1}}$ [11].

- Compound Alignment involves more than two ontologies, and contains at least one complex correspondence that involves an expression composed of named entities from two or more ontologies.

- Ternary Alignment is a special case of a compound alignment. It involves three ontologies: a source and two targets; and contains at least one complex equivalence correspondence between a named entity from the source ontology and an expression involving two named entities from the two target ontologies.

In the literature, a simple pairwise non-oriented ontology alignment is called, in short, an alignment.

${ }^{2}$ http://alignapi.gforge.inria.fr/format.html

$\sqrt[3]{\text { http://alignapi.gforge.inria.fr/edoal.html }}$ 


\subsubsection{Alignment Cardinality/Multiplicity}

The notion of alignment multiplicity is often used in the case of two matched ontologies. It describes how many entities from one ontology can be matched to an entity from another ontology [17]. Alignments can have different cardinalities. Usual notations are one-to-one $(1: 1)$, one-to-many $((1: m)$ or $(1: *))$, many-to-one $((n: 1)$ or $(*: 1))$, and many-to-many $((n: m)$ or $(*: *))$, where $*$ means zero or more entities. Alignments are usually partial alignments (i.e., not total alignments); that is there could be many entities in both ontologies that have no correspondence in the other ontology. As a result, alignments' cardinalities can rather have the following notations: (? : ?), (? : *), (*: ?) and $(*: *)$, where ? means zero or one entity. In general, alignments between independently developed ontologies are many-to-many alignments, where zero or more entities from the first ontology can match with zero or more entities from the second ontology.

Ambiguous Alignment [17] contains some correspondences that share a common entity: It matches the same entity from one ontology with several entities from the other ontology. In other words, an ambiguous pairwise alignment contains multiple correspondences having in common a source entity or a target entity. Therefore, alignments of cardinalities $(1: *)$ or $(1: m),(*: 1)$ or $(n: 1)$, and $(*: *)$ or $(n: m)$ are actually ambiguous alignments. In contrast, in one-to-one alignments $(1: 1)$, an entity (i.e., a source or target entity) appears in at most one correspondence.

\subsection{Alignment Correspondence}

A semantic correspondence is also called a mapping, a match, a relationship or a relation by some authors, although these terms are not preferred. Commonly, given two matched ontologies $O_{1}$ and $O_{2}$, a correspondence is a 5-tuple $<i d, e_{O_{1}}, e_{O_{2}}, r, n>$ [17] that can be denoted $<e_{O_{1}} r e_{O_{2}}>$, such that:

- $i d$ is the unique identifier of the correspondence.

- $e_{O_{1}}$ and $e_{O_{2}}$ are the members of the correspondence. $e_{O_{1}}$ is an entity belonging to $O_{1}$, and $e_{O_{2}}$ is an entity belonging to $\mathrm{O}_{2}$.

- $r$ is a binary semantic relation holding or intended to hold between $e_{O_{1}}$ and $e_{O_{2}}$, such as equivalence ( $\left.\equiv\right)$, subsumption $(\sqsubseteq / \sqsupseteq)$, disjointness $(\perp)$, instantiation, overlap $(\zeta)$, or named relations, etc.

- $n$ is a confidence measure assigning a degree of trust on the identified relation. It is a real number that is generally normalized in the interval $[0,1]$ to reflect the degree of truth/correctness/reliability of the correspondence. In the equivalence case, $n$ indicates whether the two entities have a high or low similarity degree. The correspondence asserts that the relation $r$ links $e_{O_{1}}$ and $e_{O_{2}}$ with a confidence value equal to $n$ and ranging between [0,1]. The higher the confidence degree, the more likely the relation holds [17].

In the crisp alignments, the confidence values of all correspondences are equal to 1.0. Following the preceding matching types, we distinguish eight correspondence types:

- Simple Correspondence involves a named entity in all of its members. A named entity can be a named concept, a named property, or a named individual. For example, if it is a pairwise correspondence, $e_{O_{1}}$ and $e_{O_{2}}$ are simple named entities. The following example is a simple pairwise correspondence written in DL and FOL languages, respectively:

$$
\begin{gathered}
O_{1}: \text { Person } \equiv O_{2}: \text { Human } \\
\forall x, O_{1}: \text { Person }(\mathrm{x}) \equiv O_{2}: \text { Human }(\mathrm{x})
\end{gathered}
$$

- Complex Correspondence involves an anonymous entity in at least one of its members, e.g. in $e_{O_{1}}$ or $e_{O_{2}}$ or both, if it is a pairwise correspondence. An anonymous entity is constructed by a logical formulation. The following example is a complex pairwise correspondence written in DL and FOL languages, respectively:

$$
\begin{gathered}
O_{1}: \text { Accepted_Paper } \equiv O_{2}: \text { Paper } \sqcap \exists O_{2}: \text { hasDecision.Acceptance } \\
\forall \mathrm{x}, O_{1}: \text { Accepted_Paper }(\mathrm{x}) \equiv \exists \mathrm{y}, O_{2}: \operatorname{Paper}(\mathrm{x}) \wedge O_{2}: \text { hasDecision }(\mathrm{x}, \mathrm{y}) \wedge O_{2}: \text { Acceptance }(\mathrm{y})
\end{gathered}
$$


- Pairwise Correspondence is a binary correspondence between two ontologies, denoted as $c=\left\langle e_{O_{1}}, e_{O_{2}}\right\rangle$, such that $e_{O_{1}}$ belongs to $O_{1}$ and $e_{O_{2}}$ belongs to $O_{2}$. The following example [32] is a complex pairwise correspondence written in DL and FOL languages, respectively:

$$
\begin{gathered}
O_{1} \text { :Professor } \equiv O_{2} \text { :AsstProf } \sqcup O_{2} \text { :AssocProf } \sqcup O_{2}: \text { FullProf } \\
\forall \mathrm{x}, O_{1} \text { :Professor }(\mathrm{x}) \equiv O_{2} \text { :AsstProf }(\mathrm{x}) \vee O_{2} \text { :AssocProf }(\mathrm{x}) \vee O_{2}: \text { FullProf }(\mathrm{x})
\end{gathered}
$$

- Holistic Correspondence, also called $N$-ary correspondence [17] or clique [36], stands for a correspondence between multiple ontologies, denoted as $c=<e_{O_{1}}, e_{O_{2}}, \ldots, e_{O_{N}}>$, such that each $e_{O_{j}}$ belongs to an ontology $O_{j}$ and $N>2$. It can only be an equivalence or disjointness correspondence. In the sake of completeness of the alignment, it could be observed that the holistic correspondence does not need to necessarily contain an entity from each ontology. Some components $\left(e_{O_{j}}\right)$ of the holistic correspondence may be empty. In other terms, a holistic correspondence should be composed of at least two entities, not necessarily exactly $N$ entities. Because when trying to only find correspondences composed of $N$ entities, other correspondences that are not shared among all input ontologies can be missed. The following example [36] is a simple holistic correspondence expressed in DL and FOL languages, respectively:

$$
\begin{gathered}
O_{1}: \text { writePaper } \equiv O_{2}: \text { writes } \equiv O_{3} \text { :hasRelatedPaper } \\
\forall \mathrm{x}, O_{1}: \operatorname{writePaper}(\mathrm{x}) \equiv O_{2}: \operatorname{writes}(\mathrm{x}) \equiv O_{3}: \text { hasRelatedPaper }(\mathrm{x})
\end{gathered}
$$

- Oriented/Directional Correspondence is a directed or ordered correspondence oriented from a source ontology to a target ontology. It is a one-way or injective function [2] relating two entities, such as subsumption relations or named (not built-in) relations. In a directional correspondence, $\left\langle e_{O_{1}} r e_{O_{2}}\right\rangle$ is not the inverse of $<e_{O_{2}} r e_{O_{1}}>$ [41]. The following example is a simple oriented pairwise correspondence in DL and FOL languages, respectively:

$$
\begin{gathered}
O_{1}: \text { Man } \sqsubseteq O_{2}: \text { Person } \\
\forall \mathrm{x}, O_{1}: \operatorname{Man}(\mathrm{x}) \sqsubseteq O_{2}: \text { Person }(\mathrm{x})
\end{gathered}
$$

- Non-Oriented/Bidirectional Correspondence is an undirected or unordered correspondence oriented in both directions: from a source to a target ontology, and vice versa. It is a two-way or bijective function [2] relating two entities, such as equivalence or disjointness relations. When a correspondence is bidirectional, $\left\langle e_{O_{1}} r e_{O_{2}}>\right.$ is the inverse of $<e_{O_{2}} r e_{O_{1}}>$.

- Compound Correspondence involves a complex compound entity in at least one of its members. A complex compound entity is an entity constructed by a logical formulation using named entities coming from two or more input ontologies.

- Ternary Correspondence involves a named entity in one member and a complex compound entity in the other member. The named entity comes from one input ontology. The complex compound entity is a logical formulation constructed by two named entities coming from two other input ontologies. The following example [32, 39] is a ternary correspondence written in DL and FOL languages:

$$
\begin{gathered}
O_{1}: \operatorname{aorticStenosis} \equiv O_{2}: \operatorname{aorta} \sqcap O_{3}: \text { constricted } \\
\forall \mathrm{x}, O_{1}: \operatorname{aorticStenosis}(\mathrm{x}) \equiv O_{2}: \operatorname{aorta}(\mathrm{x}) \wedge O_{3}: \text { constricted }(\mathrm{x})
\end{gathered}
$$

In the literature, a simple pairwise bidirectional alignment correspondence is briefly called a correspondence. Table 1 summarizes different types of ontology matching, ontology alignment, and alignment correspondence. 


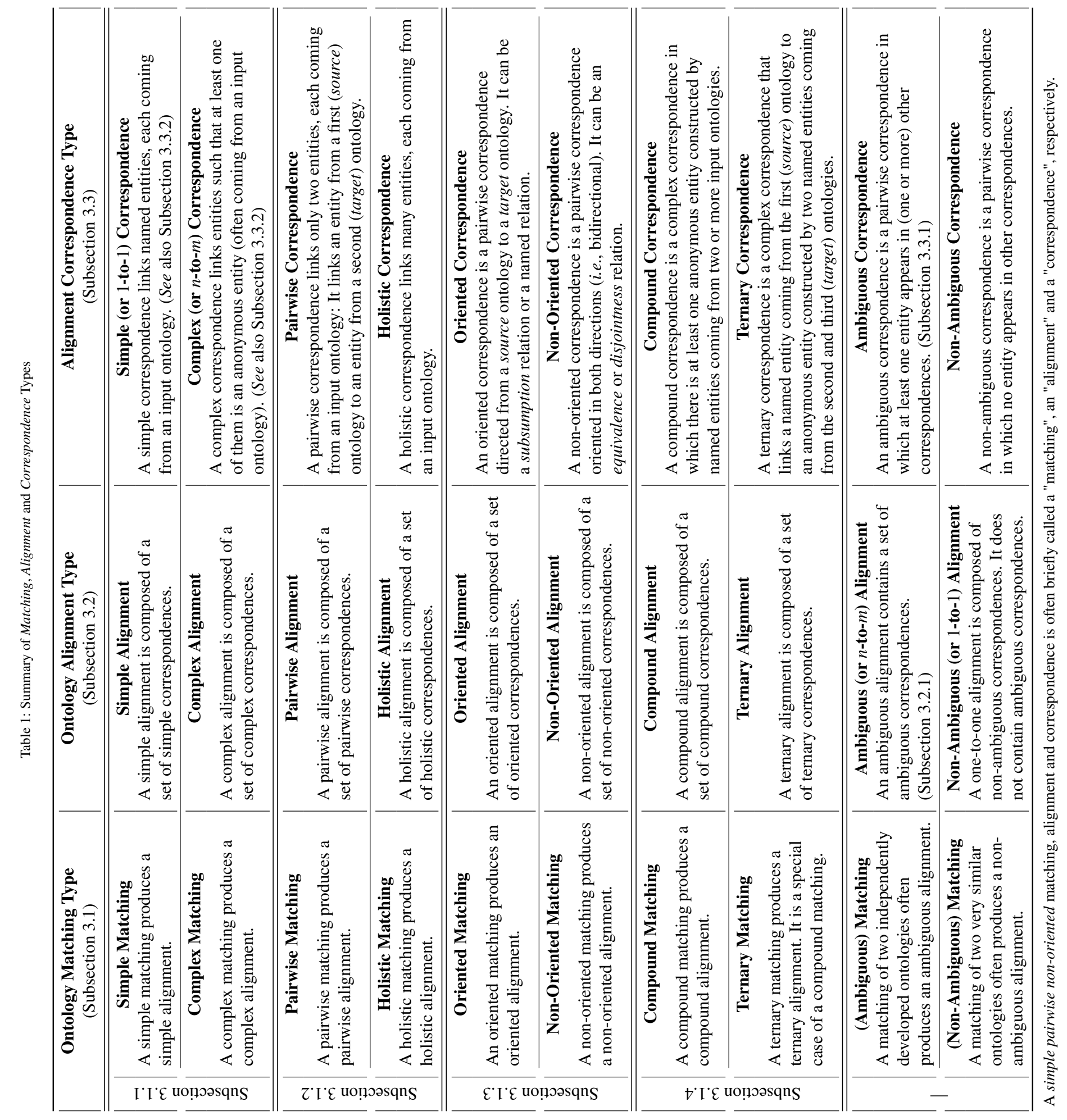




\subsubsection{Ambiguous Correspondence}

Following the preceding definition of an ambiguous alignment, we introduce the definition of an ambiguous correspondence [17], a.k.a. a correspondence of higher multiplicity [42] or a higher-multiplicity correspondence [42] It is a correspondence in which at least one member (i.e., one entity) is also involved in other correspondences. In the ambiguous pairwise alignment case, a source or target entity occurs in at least two correspondences. Ambiguous pairwise correspondences match the same entity from a first ontology with more than one entity from a second ontology. The following example shows three ambiguous correspondences in DL:

$$
\begin{gathered}
O_{1}: \text { Student } \equiv O_{2}: \text { Student } \\
O_{1}: \text { Student } \equiv O_{2}: \text { Scholar } \\
O_{1}: \text { Student } \equiv O_{2}: \text { PhD_Student }
\end{gathered}
$$

\subsubsection{Correspondence Cardinality/Multiplicity}

The notion of correspondence multiplicity is different from the notion of alignment multiplicity (See Subsection 3.2.1. This notion is also often used in the case of two matched ontologies. It describes how many entities from each ontology are involved in one correspondence (not in a whole alignment). It reflects the correspondence complexity. Correspondences can have different cardinalities 4 Usual notations are one-to-one $(1: 1)$ for the simple correspondences; and one-to-many $((1: m)$ or $(1: *))$, many-to-one $((n: 1)$ or $(*: 1))$ and many-to-many $((n: m)$ or $(*: *))$ for the complex correspondences, where $*$ means zero or more entities.

\subsection{Ontology Mapping}

In the literature, the term mapping can correspond to either a matching process, an alignment file, or a correspondence. Indeed, mapping can be used as a noun and a verb in the "-ing" form, which can be confusing. It is better to avoid it and use instead the aforementioned terms. However, some authors try to propose a standard definition for the term mapping. Faria et al. [43] defined a mapping as an equivalence correspondence. Whereas Euzenat and Shvaiko [17] defined it as a non-ambiguous oriented/directed equivalence alignment, containing oriented/directional equivalence correspondences that can be called mapping rules once interpreted as ontological statements/axioms.

\subsection{Correspondence Relation}

A semantic relation of a given correspondence can be a built-in relation such as the equivalence relation ( $\equiv)$, the subsumption relation $(\sqsupseteq$ or $\sqsubseteq)$, the disjointness or incompatibility relation $(\perp)$, and the instantiation relation (i.e., has instance or instance of). In an alignment, relations can be flagged by the following symbols: "=" (i.e. is equivalent to), ">" (i.e. subsumes or is more general than), "<" (i.e. is subsumed by or is more specific than), and "\%" (i.e. incompatible with). A semantic relation is not only restricted to built-in/predefined relations of the OWL ontology language, but also includes other relations such as the overlap relation $(\zeta$ or $\sqcap)$, named relations, and fuzzy relations. In the following, we describe the three most common built-in relations:

- Equivalence Relation: An equivalence relation holding between two classes $A$ and $B$ means that all instances of $A$ are also instances of $B$, i.e. both classes contain exactly the same set of individuals as instances. An equivalence relation holding between two properties $P_{1}$ and $P_{2}$ means that an individual $x$ can be connected to an individual or a data literal $y$ by both $P_{1}$ and $P_{2}$. An equality relation holding between two individuals $x$ and $y$ means that the individual $x$ is identical/equal/synonymous to the individual $y$ and refers to the same thing.

- Subsumption Relation: A subsumption relation holding between two classes $A$ and $B$ means that the set of instances of $A$ is a subset/superset of the set of instances of $B$. A subsumption relation holding between two properties $P_{1}$ and $P_{2}$ means that if an individual $x$ is connected by $P_{1}$ to an individual or a data literal $y$, then $x$ is also connected by $P_{2}$ to $y$.

\footnotetext{
${ }^{4}$ Notations of multiplicity are introducing ambiguity in the literature because multiplicity notations of both alignments and correspondences are the same. Therefore, in every mention of the word multiplicity, we precise whether it is an "alignment" multiplicity or a "correspondence" multiplicity.
} 
- Disjointness Relation: A disjointness relation holding between two classes $A$ and $B$ means that instances of $A$ are definitely not instances of $B$. In other words, no individual $x$ can be at the same time, an instance of both $A$ and $B$. A disjointness relation holding between two properties $P_{1}$ and $P_{2}$ means that no individual $x$ can be connected to an individual or a data literal $y$ by both $P_{1}$ and $P_{2}$. An inequality relation holding between two individuals $x$ and $y$ means that the individual $x$ cannot be identical/equal/synonymous to $y$ and does refer to a different thing.

According to Cheatham and Pesquita [32], the simplest types of relations are equivalence and disjointness, and then comes the subsumption relation. However, Solimando et al. [44] consider equivalence and subsumption to be the simplest relations, and then comes the disjointness relation, because negative constraints are typically harder to identify and assess than positive ones. That is why most of the available matching systems do not compute disjointness relations. Arbitrary relations are even harder to find. An arbitrary relation [32] is any type of relations: equivalence, subsumption, disjointness, etc. Consequently, the simplest type of ontology matching is the one that finds simple pairwise equivalence correspondences, which is the most common ontology matching type. Then, the one that finds simple pairwise subsumption and disjointness correspondences comes next in the difficulty level.

\subsection{Network of Ontologies}

Denoted as $\langle\Omega, \Lambda>$, a network of ontologies [17], a.k.a. a network of aligned ontologies [45] or networked ontologies [46], is composed of a set of ontologies $\Omega$ and a set of pairwise alignments $\Lambda$ between arbitrary pairs of these ontologies. It constitutes a sort of distributed ontology [47].

A normalized network of ontologies [17], denoted as $\langle\Omega, \Lambda\rangle$, is a network of ontologies with exactly one alignment between each possible pair of its ontologies. In other words, a network of ontologies is called a normalized one, if and only if $\left|\Lambda\left(O, O^{\prime}\right)\right|=1$ for any two ontologies $O$ and $O^{\prime}$ composing it, where $\Lambda\left(O, O^{\prime}\right)$ denotes the set of existing alignments between $O$ and $O^{\prime}$.

\section{Ontology Integration}

In this section, we provide some of the consensual definitions of the notions of ontology integration and ontology merging in the literature and describe their different existing types.

\subsection{Ontology Integration vs. Ontology Merging}

Ontology integration and merging terms are ambiguous. Some authors consider and use both of them as synonyms, whereas other authors do not. In the remainder, we will refer to these terms as defined in this subsection.

In general, ontology integration/merging is the process of integrating/merging two or more ontologies into a single one [48]. However, more precisely, ontology integration or merging is the process of reusing or unifying existing ontologies to build a new more general or more complete one that can be utilized by a specific application or by existing applications already using the input ontologies that were integrated or merged [4-6]. Ontology merging is sometimes referred to as ontology fusion [4-6, 49].

Euzenat and Shvaiko [17] defined ontology merging by the process of creating a new ontology from two or more, possibly overlapping, source ontologies. As for the integration, they stated that ontology integration is the inclusion, in an ontology, of another ontology along with links between these two ontologies, as prescribed in the alignment between them. The only difference between these two definitions is that, unlike ontology merging which does not alter the input ontologies in the merging process, ontology integration alters one of the input ontologies-the target ontology - and keeps the other source ontologies unaltered. The authors [17] formalized the merging of two ontologies by a merge operator, $\operatorname{Merge}\left(O_{1}, O_{2}, \mathcal{A}\right)=O_{3}$, where $O_{1}$ and $O_{2}$ are the source ontologies to be merged, and $\mathcal{A}$ is the alignment expressed in the same logical language as ontologies $O_{1}$ and $O_{2}$. As for the ontology integration, we suggest the integration operator, Integrate $\left(O_{1}, O_{2}, \mathcal{A}\right)=O_{1}$, where $O_{1}$ is the target ontology into which the source ontology $O_{2}$ will be integrated.

We believe that the ontology integration notion is synonymous to the ontology enrichment, inclusion or extension notions, and that it also includes the merging notion. In other words, we consider merging as a special case of integration. In fact, the result of including/integrating source ontologies into an empty target ontology is equivalent to the result 
of merging them. For example, if it is about two input ontologies $O_{1}$ and $O_{2}$, the resulting ontology will be $O_{1}$ in the integration case, and will be $\mathrm{O}_{3}$ in the merging case. Therefore, merging ontologies can be considered as integrating ontologies into one another. More simply, merging two ontologies $O_{1}$ and $O_{2}$ can be considered as simultaneously integrating $O_{1}$ into $O_{2}$ and $O_{2}$ into $O_{1}$ (in both directions). Consequently, a resulting merged ontology can be called an integrated ontology. Hence, we have chosen to use the term integration in the title and all the remaining, rather than merging. In Table 2, we summarize the differences between definitions of ontology integration and ontology merging.

Table 2: Description of Ontology Merging and Ontology Integration

\begin{tabular}{|c|c|c|c|c|c|}
\hline & \multicolumn{2}{|c|}{ Ontology Merging } & \multicolumn{3}{|c|}{ Ontology Integration } \\
\hline $\begin{array}{l}\text { Integration } \\
\text { Type }\end{array}$ & \multicolumn{2}{|c|}{ Simple Merge, or } & \multicolumn{3}{|c|}{\begin{tabular}{|c|l|} 
Simple Merge, & Full Merge, \\
or Asymmetric Merge
\end{tabular}} \\
\hline Final Goal & \multicolumn{2}{|c|}{ To build a brand new ontology } & \multicolumn{3}{|c|}{ To enrich and extend an ontology } \\
\hline Method & \multicolumn{2}{|c|}{ Assembling/Aggregation } & \multicolumn{3}{|c|}{ Inclusion } \\
\hline $\begin{array}{l}\text { Method } \\
\text { Details }\end{array}$ & \multicolumn{2}{|c|}{$\begin{array}{l}\text { Assembling source ontologies } \\
\text { to form a target } \text { ontology }\end{array}$} & \multicolumn{3}{|c|}{$\begin{array}{c}\text { Incorporating source ontologies -one by one- } \\
\text { into a target ontology }\end{array}$} \\
\hline Linkage* $^{*}$ & \multicolumn{2}{|c|}{ Weak or strong } & \multicolumn{3}{|c|}{ Weak or strong } \\
\hline $\begin{array}{l}\text { Input } \\
\text { Ontologies }\end{array}$ & \multicolumn{2}{|c|}{2 or more source ontologies } & \multicolumn{3}{|c|}{$\begin{array}{c}1 \text { target ontology }+ \\
1 \text { or more source ontologies }\end{array}$} \\
\hline $\begin{array}{l}\text { Output } \\
\text { Ontology }\end{array}$ & \multicolumn{2}{|c|}{$\begin{array}{l}\text { A newly built target ontology, } \\
\text { called a "merged" ontology }\end{array}$} & \multicolumn{3}{|c|}{$\begin{array}{l}\text { A new version of the target ontology, } \\
\text { called an "integrated" ontology }\end{array}$} \\
\hline Results & \multicolumn{2}{|c|}{$\begin{array}{l}\text { The source ontologies are } \\
\text { replaced by the "merged" ontology }\end{array}$} & \multicolumn{3}{|c|}{$\begin{array}{l}\text { The target ontology is replaced/overwritten } \\
\text { by the "integrated" ontology }\end{array}$} \\
\hline $\begin{array}{l}\text { Integration } \\
\text { Purpose }\end{array}$ & $\begin{array}{c}\text { To } \\
\text { interoperate } \\
\text { applications } \\
\text { already using } \\
\text { the source } \\
\text { ontologies }\end{array}$ & $\begin{array}{l}\text { To build } \\
\text { an ontology } \\
\text { in order to } \\
\text { be used by } \\
\text { a particular } \\
\text { application }\end{array}$ & $\begin{array}{l}\text { To enrich } \\
\text { an ontology } \\
\text { already } \\
\text { used } \\
\text { by existing } \\
\text { applications }\end{array}$ & $\begin{array}{l}\text { To enrich an } \\
\text { ontology under } \\
\text { construction, } \\
\text { to be used by } \\
\text { a particular } \\
\text { application }\end{array}$ & $\begin{array}{c}\text { To enrich } \\
\text { an empty } \\
\text { ontology, } \\
\text { which leads } \\
\text { to ontology } \\
\text { merging }\end{array}$ \\
\hline $\begin{array}{l}\text { Source } \\
\text { Ontologies' } \\
\text { Knowledge } \\
\text { Preservation }\end{array}$ & Mandatory & $\begin{array}{l}\text { Not } \\
\text { mandatory }\end{array}$ & $\begin{array}{l}\text { Not } \\
\text { mandatory }\end{array}$ & $\begin{array}{l}\text { Not } \\
\text { mandatory }\end{array}$ & $\begin{array}{l}\text { See the } \\
\text { ontology } \\
\text { merging } \\
\text { column }\end{array}$ \\
\hline $\begin{array}{l}\text { Target } \\
\text { Ontology's } \\
\text { Knowledge } \\
\text { Preservation }\end{array}$ & - & - & Mandatory & $\begin{array}{l}\text { Not } \\
\text { mandatory }\end{array}$ & $\begin{array}{l}\text { See the } \\
\text { ontology } \\
\text { merging } \\
\text { column }\end{array}$ \\
\hline $\begin{array}{l}\text { Domains } \\
\text { of the Input } \\
\text { Ontologies }\end{array}$ & $\begin{array}{l}\text { Same/Similar } \\
\text { domains }\end{array}$ & $\begin{array}{c}\text { Same/Related } \\
\text { or different } \\
\text { domains }\end{array}$ & $\begin{array}{c}\text { Same/Similar } \\
\text { domains }\end{array}$ & $\begin{array}{c}\text { Same/Related } \\
\text { or different } \\
\text { domains }\end{array}$ & $\begin{array}{l}\text { See the } \\
\text { ontology } \\
\text { merging }\end{array}$ \\
\hline
\end{tabular}

* Weak: equivalent entities are simply linked by equivalence axioms.

* Strong: equivalent entities are completely merged to constitute a single entity.

\subsection{Types of Ontology Integration}

We distinguish three distinct ontology integration approaches derived from our extensive state-of-the-art research, namely: (i) the simple merge (or the bridge ontology) and (ii) the full merge, which are semantically equivalent; and (iii) the asymmetric merge which is actually an ontology enrichment. Only the targeted application and usage can 420 justify preferring one integration approach over another. 


\subsubsection{Simple Merge}

The first approach is the Simple Merge, a.k.a. the Reduced Semantics [17] or the Simple Union [27]. It imports the input ontologies into a new ontology — constituting a union of input ontologies—and adds bridge/bridging/articulation axioms translating the alignment between them (See Figure 1a). These added axioms are actually semantic correspondences interpreted as or transformed into ontological statements to link/connect the overlapping part of the input ontologies. They are called a semantic bridge [50], articulations, or reductionistic alignment semantics [51] (abbreviated as alignment semantics or reductionistic semantics). The reductionistic alignment semantics is so called, because the semantics of an alignment is reduced to the semantics of Description Logics, thus to a set of axioms. The correspondences of the alignment $\mathcal{A}$ can be perceived as an ontology $O_{\mathcal{A}}$ called (semantic) bridge ontology [1, 2, 52], articulation ontology [50], articulation [50], intersection ontology [53], or intermediate ontology [11]. Following the recommendation of the $\mathrm{W} 3 \mathrm{C}$ best practices group [54], most of the state-of-the-art approaches assume that the input ontologies are in OWL and correspondences between them are also interpreted as OWL axioms:

- The subsumption, equivalence, and disjointness correspondences between classes are expressed by built-in subClassOf, equivalentClass and disjointWith OWL axioms, respectively;

- The correspondences between properties are expressed by built-in subPropertyOf, equivalentProperty and propertyDisjointWith OWL axioms, respectively; and

- The equality and inequality correspondences between individuals are expressed by built-in sameAs and differentFrom OWL axioms, respectively.

- Whereas the identifiers and the confidence values of the correspondences are expressed by OWL annotations, which are non-logical axioms having no effect on inferences [55].

Therefore, in the case of two input ontologies, the integrated ontology $O_{3}$ can be viewed as the union of $O_{1}, O_{2}$ and $O_{\mathcal{A}}$ where $O_{3}=O_{1} \cup O_{2} \cup O_{\mathcal{A}}$ [55]. In this type of merge, equivalent entities are mentioned more than once in the integrated ontology but linked by equivalence axioms, hence they can be considered as non-redundant entities (See Figure 1a. It should be noted that correspondences can be interpreted in other languages 5 other than OWL, such as SWRL (Semantic Web Rule Language) [59] or SKOS (Simple Knowledge Organization System) [60]. The resulting ontology can be called an aligned ontology [44, 61], a merged ontology or an integrated ontology.

To achieve ontology modularization, the OWL language provides a built-in import statement $<$ owl:imports $>$. The latter includes the content of an entire ontology into the current ontology by only referencing the URI or the local file of that ontology. Therefore, the integrated ontology $O_{3}$ is generally obtained by converting the RDF alignment to an OWL ontology $O_{A}$ which directly imports the two input ontologies $O_{1}$ and $O_{2}$; so that $O_{A}$ becomes $O_{3}$. Otherwise, the integrated ontology $O_{3}$ is obtained by creating an empty ontology that directly imports $O_{1}, O_{2}$ and $O_{A}$. The import is automatically performed by simply declaring the OWL import statements referencing the ontologies to be imported. In this case, the resulting ontology $O_{3}$ is generally called a bridge ontology.

\subsubsection{Full Merge}

The second approach is the Full Merge [27], a.k.a. the Complete Merge [1, 2] or the Symmetric Merge [27]. It imports the input ontologies into a new ontology — constituting a union of input ontologies—and merges/combines each set of equivalent entities into a single new entity that preserves all their attached description and relations (See Figure $1 \mathrm{~b}$. Ontological axioms, constituting the merged ontology and originating from the input ontologies, are updated by replacing every occurrence of the original entities with its new merged entity. That is, each axiom, in which appears the name of one of the entities that have been merged, must be updated by replacing the name of that original entity with the name of the newly merged one. Subsumption axioms can also be added to link subsuming and subsumed entities, as prescribed in the alignment(s).

In the literature, authors identify the merged entities by either a unique (alphanumeric) code or by the name of one of the original entities that have been merged - commonly, by the name of the entity that belongs to the preferred

\footnotetext{
${ }^{5}$ Some other languages or formalisms have been proposed and used in the literature for expressing the semantic correspondences, such as C-OWL (Contextualized OWL) [56], DDL (Distributed Description Logics) [57] and $\varepsilon$-connected OWL [58] that are designed to reason on ontologies connected by directional alignments, i.e. to reason on a network of aligned ontologies, not in a merged ontology as in our case.
} 
input ontology; then, they add the short names of the original entities (that have been merged) as additional labels to the newly merged entity. The merged entities will be represented only once, which avoids the existence of redundant entities in the merged ontology. In the case of two input ontologies, the merged ontology $\mathrm{O}_{3}$ can be viewed as the union of $O_{1}$ and $O_{2}$ where $O_{3}=O_{1} \cup O_{2}=\left(O_{1}-O_{2}\right) \cup\left(O_{2}-O_{1}\right) \cup\left(O_{1} \cap O_{2}\right)$ [1]. Multiple inheritance, resulting from assigning more than one direct parent to each merged entity, will generate multiple root is- $a$ paths to the entities of the merged ontology. The resulting ontology can be referred to as a unified ontology [4, 50, 62], a merged or an integrated ontology.

After choosing an input ontology as a transforming ontology, a full merge can also be performed by applying an ontology transformation process to $N-1$ input ontologies, using pairwise alignments between the transforming ontology and each of the $N-1$ ontologies to be transformed. Then, an ontology composition/aggregation process is applied to all of the $N$ input ontologies (i.e., the transforming ontology and the $N-1$ transformed ones) [17]. For instance, in the case of two ontologies $O_{1}$ and $O_{2}$ and an alignment $\mathcal{A}$ between them, entities of $O_{2}$ are expressed by equivalent entities of $O_{1}$ (or inversely) as mentioned in the alignment $\mathcal{A}$, then $O_{1}$ and $O_{2}$ are aggregated to constitute the merged ontology $O_{3}$. Therefore, $O_{3}=O_{1} \cup O_{2}^{\prime}$ such that $O_{2}^{\prime}=\operatorname{Transform}\left(O_{2}, \mathcal{A}\right)$.

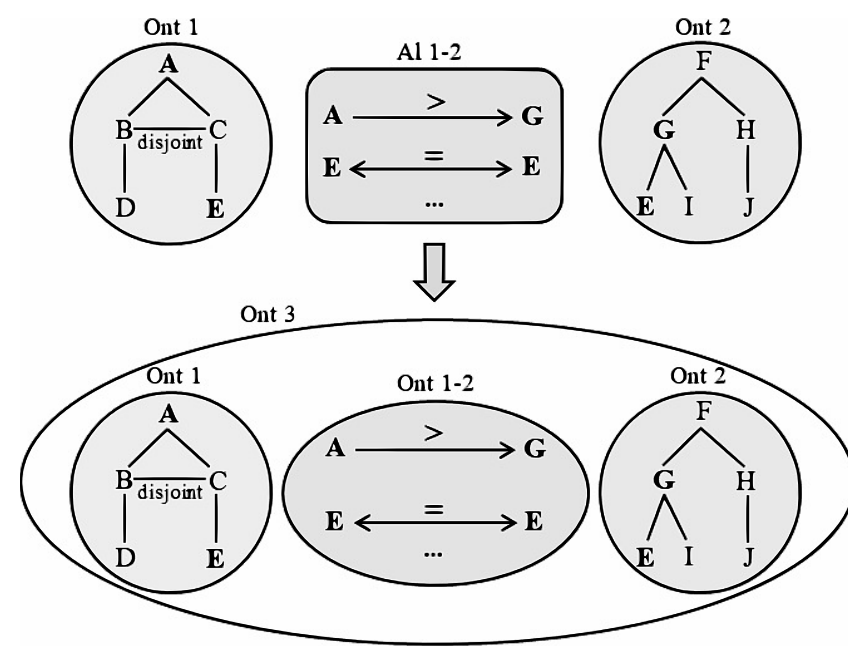

(a) Simple-Merge Ontology

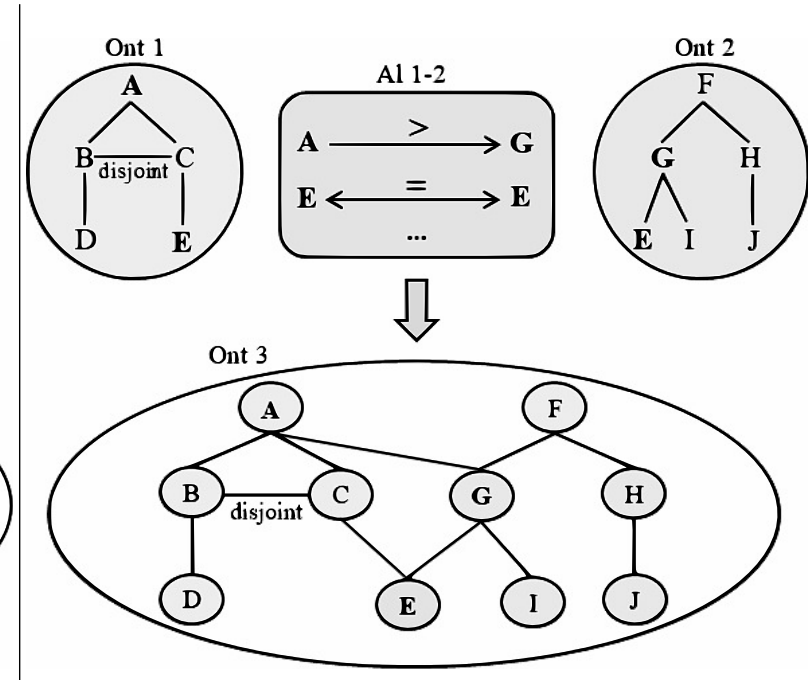

(b) Full-Merge Ontology

Figure 1: Ontology Integration/Merging Types

\subsubsection{Asymmetric Merge}

The third approach is the asymmetric merge [63, 64], a.k.a. the target-driven asymmetric merge, the ontology enrichment [42] or the ontology integration [42]. It takes one of the input ontologies as the target ontology into which the other source ontologies will be incrementally integrated to enrich/extend it. The merging manner can follow the full merge approach or the simple merge approach.

The target ontology is the priority (preferred) ontology, whereas the source ontologies have a lower priority. The target ontology is also called the seed ontology [42], the backbone ontology [42] or the knowledge base [8, 42]. The latter should be completely preserved during the integration process because it may already be in use by various applications or services. Thus, all of its axioms (especially its structure) should not be altered, and all of its entities should be preserved. However, entities and axioms (including the structure) of the source ontologies can be removed or modified if they are redundant or in disagreement with the target ontology conceptualization. In this respect, we believe that this asymmetric merge is actually an integration or an enrichment of the target ontology using merging techniques, rather than a merge. The resulting ontology is called an integrated ontology. 


\section{Ontology Integration Principles, Violations and Repair}

In this section, we explain the principles that an ontology integration should fulfill, the reasons behind the emergence of errors (or issues) in the integrated ontologies, and the repair strategies applied to resolve these errors.

\subsection{Ontology Integration Principles (and Examples of their Violation)}

Integrating ontologies using alignments can lead to unintended consequences such as semantic conflicts or contradictions, redundancies and cycles, etc. Pottinger and Bernstein [65] proposed a set of requirements for merging models such as database schemata, ontologies, and UML models, etc. However, these merge requirements are very generic and need to be specialized for the case of ontology merging (and integration). In this paper, we have gathered the most relevant requirements (or principles) that an ontology integration should try to fulfill, and they are as follows:

\section{Integrated Ontology Requirements}

(a) Alignment correctness and completeness,

(b) Ontology knowledge preservation,

i. Entities preservation,

ii. Axioms/Entailments preservation,

(c) Alignment correspondences preservation,

(d) Ontology coherence (and consistency),

(e) Ontology conservativity,

\section{General Ontology Requirements}

(a) Minimality (or prohibition of redundant entities),

(b) Acyclicity (or prohibition of subsumption cycles),

(c) Prohibition of structural and relational redundancy,

(d) Property's domain and range oneness,

(e) Entity connectivity (or prohibition of unconnected entities), etc.

We distinguish the specific requirements of an integrated ontology and the general requirements of any ontology. It is impossible to simultaneously satisfy all the ontology integration requirements, but they should be satisfied as much as possible. We have added a requirement that we have called the prohibition of structural and relational redundancy. The latter, as well as the principles (a), (b), (d) and (e), are requirements that any ontology should preferably fulfill (not particularly an integrated one). In the following, we explain each principle/requirement in a separate subsection, and we support them with some violation examples.

\subsubsection{Alignment Correctness and Completeness Principle}

This feature only depends on the performance of the ontology matching step. Ontology matching tools may either lack correct correspondences, contain incorrect correspondences, or both. Ideally, the ontology alignment should not contain any false correspondences, which reflects the alignment correctness, and should contain all the correct correspondences, which reflects the alignment completeness.

\subsubsection{Ontological Knowledge Preservation Principle}

The principle of knowledge preservation, a.k.a. knowledge completeness or knowledge coverage [27], is the preservation of all entities and axioms from the input ontologies that were integrated. Entities or elements include all classes (i.e., class preservation), properties (i.e., property preservation) and individuals (i.e., instance preservation) from the input ontologies. Axioms include all kinds of triplets such as subsumption, equivalence and disjointness axioms, annotations, declarations, expressions, restrictions and assertions from the input ontologies; not only subsumption axioms of their class hierarchies (i.e., relationship preservation [65] or structure preservation). Indeed, all entailments (i.e., inferred axioms) of the input ontologies should be preserved in the integrated ontology [55]. Hence, the knowledge preservation principle is composed of two sub-principles: (i) the entity/element preservation principle [65], and (ii) the axiom preservation principle, also called the entailment deduction satisfaction [66]. The knowledge preservation principle applies to cases of simple-merge and full-merge ontology integration. However, in the case of asymmetric merge, it applies only on the target/priority ontology. Still, the knowledge of the source/non-priority ontologies should be preserved as much as possible in the integrated ontology. 


\subsubsection{Alignment Preservation Principle}

\subsubsection{Ontology Coherence Principle}

Before defining the coherence principle, let us clarify some ambiguous related notions. The three following terms are used in the literature in a confusing manner: (un)satisfiability, (in) consistency and (in)coherence.

Satisfiability/Unsatisfiability [67]. This notion is related to entities (classes and properties). An unsatisfiable entity is requirements to be a member of that entity. Recall that a class is instantiated by individuals; and a property is instantiated by pairs of individuals (and data literals): an individual in the property domain, and an individual or a data literal in the property range. An unsatisfiable class can (and should) never have instances, like does the class owl:Nothing, because there will be no instance that can satisfy it [67]. Similarly, an unsatisfiable property can (and should) never ave instances, because there will be no instance that can satisfy it, like do the properties owl:bottomObjectProperty and owl:bottomDataProperty.

Consistency/Inconsistency [68]. This notion is related to ontologies. An ontology is consistent if it has a satisfying interpretation. For example, an ontology from which we deduce that an individual $x$ is different from and identical to an individual $y$, cannot have a satisfying interpretation. Inconsistency can occur whenever there is at least one violation of a class restriction, one instantiation of an unsatisfiable class or property, one instantiation of two disjoint classes or properties, or one semantic contradiction between individuals in the A-box like in the previous example, etc. Since an inconsistent ontology has no model, all of its classes and properties become unsatisfiable, i.e. none of its classes and properties can be instantiated. It is considered as a severely damaged ontology, containing a serious error that must be repaired because no useful knowledge can be inferred from it by ontology reasoning. In addition, it cannot be published nor deployed in applications that require a logical reasoning process [68]

Coherence/Incoherence [67]. This notion refers to ontologies. An ontology is called coherent when all of its classes and properties are satisfiable. If there is at least one unsatisfiable class (except owl:Nothing) or one unsatisfiable property (except owl:bottomObjectProperty and owl:bottomDataProperty) in an ontology, then the latter becomes incoherent. An incoherent ontology is still consistent, thus it can be published and used in applications. However, if an inconsistency may always occur [67].

$$
\begin{aligned}
& \text { Coherence } \Rightarrow \text { Consistency } \\
& \text { Coherence } \nLeftarrow \text { Consistency }
\end{aligned}
$$

\section{Incoherence $\nRightarrow$ Inconsistency}

Incoherence $\Leftarrow$ Inconsistency

In the literature, the coherence principle is called the consistency principle. However, we will rather use the coherence term which seems to us more appropriate. The coherence principle states that all entities of the integrated ontology should be satisfiable, assuming that the input ontologies also do not contain any unsatisfiable entities [44]. In other words, it states that there is no additional unsatisfiable class or property in the integrated ontology. The coherence principle actually aims at the coherence (and the consistency) of the integrated ontology, assuming that input ontologies are coherent too.

If logical reasoning is involved in an application, then ensuring coherence becomes mandatory because the integrated ontology must be logically/semantically correct to support reasoning and be really useful. Indeed, in this case, an incoherent ontology may lead to incomplete or unexpected results, although it can be used in other applications. For example, in a text annotation application, it is not necessary to ensure coherence in the integrated ontology because the annotation task does not involve a reasoning process. However, in other applications such as query answering, logical errors may have a critical impact on the query answering process. 
Example 1 (Coherence Violation). Let us assume that we have a class $A$ in $O_{1}$, two disjoint classes $B$ and $C$ in $O_{2}$, and two correspondences $c_{1}$ and $c_{2}$ stating that $A$ is a subclass of $B$ and $C$. Formally,

$$
\begin{gathered}
O_{1}=\{A\} \quad O_{2}=\{B \perp C\} \\
\mathcal{A}=\left\{c_{1}, c_{2}\right\} \quad c_{1}=<A \sqsubseteq B>\quad c_{2}=<A \sqsubseteq C>
\end{gathered}
$$

If a reasoning process is applied to
a subclass of two disjoint classes.

Example 2 (Coherence Violation). Consider the example proposed by Fahad et al. [69]. Suppose that in $O_{1}$, we have two disjoint classes, "Employee" and "Student", and a class "PhD_Researcher" which is a subclass of "Student". While in $\mathrm{O}_{2}$, "Employee" and "Student" are not disjoint, and "PhD_Researcher" is a subclass of both "Employee" and "Student". Formally,

$$
\begin{aligned}
& O_{1}=\{\text { Employee } \perp \text { Student }, \text { PhD_Researcher } \sqsubseteq S t \text { tudent }\} \\
& O_{2}=\left\{\text { Employee }, S \text { tudent }, P h D \_ \text {Researcher } \sqsubseteq \text { Employee },\right.
\end{aligned}
$$$$
\text { PhD_Researcher } \sqsubseteq \text { Student }\}
$$

If a reasoning process is applied to the ontology $\bar{O}_{3}$, then "PhD_Researcher" will be an unsatisfiable class since it will become a subclass of two disjoint classes.

Errors such as unsatisfiable classes and unsatisfiable properties (i.e., ontology incoherence) and ontology inconsistency are very likely to occur in an integrated ontology. They are reflections of the unintended logical inferences that are still difficult to detect beforehand, understand, explain, and repair. As a consequence, an integrated ontology will always be prone to errors, which will lower its performance and make it non-understandable and even unusable.

\subsubsection{Ontology Conservativity Principle}

The principle of conservativity requires that the integrated ontology does not introduce new semantic relations between entities of each (or at least one) of the input ontologies, especially new subsumption relations causing structural changes [44, 70, 71]. In other words, the original description of an input ontology, especially the is-a structure of its class hierarchy, should not be altered after being integrated. Consequently, the behavior of the services already functioning with that input ontology is not affected by the use of the new integrated ontology.

\section{Violation of the Conservativity Principle}

The integrated ontology may contain a large number of conservativity violations, which are not limited only to classification and structural changes (if we are dealing with the unrestricted conservativity problem). Like coherence violations, conservativity violations reveal either erroneous correspondences or an incompatibility/disagreement between the input ontologies [44]. However, they may also evidence incompleteness in (one of) the input ontologies [44].

Example 3 (Conservativity Violation). Consider the following two ontologies: $O_{1}$ contains two classes $A$ and $B$; and $\mathrm{O}_{2}$ contains two classes $A^{\prime}$ and $B^{\prime}$ where $B^{\prime}$ is a subclass of $A^{\prime}$. Formally,

$$
\begin{gathered}
O_{1}=\{A, B\} \quad O_{2}=\left\{B^{\prime} \sqsubseteq A^{\prime}\right\} \\
\mathcal{A}=\left\{c_{1}, c_{2}\right\} \quad c_{1}=<A \equiv A^{\prime}>\quad c_{2}=<B \equiv B^{\prime}>
\end{gathered}
$$

Suppose that we have to integrate $O_{2}$ into $O_{1}$, that is to say that the description of $O_{1}$ should not be altered after the integration process. If the ontology matching step generates two correspondences $c_{1}$ and $c_{2}$ stating that $A$ is 
equivalent to $A^{\prime}$, and $B$ is equivalent to $B^{\prime}$, then the original structure of $O_{1}$ will change because of the addition of a new subsumption relationship linking $A$ and $B$ after a full merge.

In the following subsections, we shed light on general ontology requirements dealing with redundancy of entities, subsumption cycles, redundancy of the structure, redundancy of relations, multiplicity of property domains and ranges, and unconnected entities, etc. Although they are not problematic from a semantic/logical point of view, these issues do undoubtedly reduce the conciseness of the integrated ontology and should be better avoided in any ontology (not only in integrated ones). We can find general ontology requirements in many ontology evaluation works, e.g, [65, 66, 72, 73].

\subsubsection{Minimality Principle}

The principle of minimality [74] states that no redundant entities should appear in an integrated ontology, assuming that the input ontologies (that have been integrated) also do not contain any of them. This principle means the prohibition of entity redundancy or duplication. Entities are called redundant or duplicated whenever they do have the same meaning, and therefore, whenever they represent the same entity in an ontology.

\section{Redundancy $\mathcal{E}$ Ambiguity of Entities}

Redundancy or duplication of entities in an (integrated) ontology occurs whenever there are distinct but actually equivalent entities coming from different input ontologies. They can also have a large overlap in their terms, which makes them ambiguous entities, i.e. having a high degree of ambiguity. Ambiguity is assessed through the number of labels appearing in multiple entities of a given ontology [42]. These common entities are redundant because they are neither linked by equivalence relations, nor merged with each other in the integrated ontology. Ontology integration should not introduce additional duplication and ambiguity. Indeed, redundant entities will increase the size of the integrated ontology, complicate text annotation tasks due to ambiguity, and decrease the interoperability between applications that use these entities [42]. Indeed, a service of an application that is using a class $X$ cannot interoperate with a service of another application that is using an equivalent class $Y$, since an equivalence axiom linking these two classes is missing [42]. However, in an integrated ontology, ensuring the coherence and conservativity principles will lead to numerous redundant entities.

\subsubsection{Acyclicity Principle}

The principle of acyclicity [65, 66] or cycle prohibition states that no subsumption cycles should appear in the class or property hierarchy of an integrated ontology, assuming that the input ontologies also do not contain any of them. Subsumption cycles have the form $\left\{e_{1} \sqsubseteq e_{2}, \ldots, e_{n} \sqsubseteq e_{1}\right\}$, where $n>1$ and $e$ are entities of the same type (classes or properties) [42]. Subsumption/is- $a$ relations should be acyclic because they complicate graph-based algorithms such as path extraction, hierarchy traversal and depth counting, leading to an undetermined depth (hierarchy level) in the class or property hierarchy [42]. Consequently, ontology integration should not introduce additional cycles. However, unexpected cycles may usually appear in an integrated ontology. Figure 6 shows an illustrative example of a subsumption cycle that we will explain later in section 8

\subsubsection{Principle of Structural and Relational Redundancy Prohibition}

We propose another principle called prohibition of structural and relational redundancy. The latter states that no structural or relational redundancies should appear in an integrated ontology, assuming that the input ontologies also do not contain any of them.

\section{Structural Redundancy}

Structural Redundancy or briefly redundancy [75], a.k.a. semantic overlap [27] or semantic redundancy [27], is the redundancy that occurs in the class hierarchy, i.e., whenever there is more than one is- $a$ path from a class to a parent other than the root [75]. In other words, it happens whenever there is more than one is-a path from the ontology root to a leaf concept. These paths are called leaf paths [27]. This type of redundancy is caused by the multiple inheritance resulting from a full merge. We think that this requirement is too strict and can be relaxed, because the multiple inheritance exists in many real-world ontologies and does not necessarily lead to semantic/logical conflicts. Similarly, instance overlap [63, 64] happens whenever an individual is instantiated by more than one concept/class. 
Example 4 (Structure Redundancy). Consider two ontologies $O_{1}$ and $O_{2}$. In $O_{1}$, "PhD_Student" is a subclass of "Student". In $\mathrm{O}_{2}$, "PhD_Student" is a subclass of Researcher.

$$
\begin{gathered}
O_{1}=\{\text { PhD_Student } \sqsubseteq \text { Student }\} \quad O_{2}=\{\text { PhD_Student } \sqsubseteq \text { Researcher }\} \\
O_{3}=\{\text { PhD_Student } \sqsubseteq \text { Student }, \text { PhD_Student } \sqsubseteq \text { Researcher }\}
\end{gathered}
$$

In the fully merged ontology $\mathrm{O}_{3}$, "PhD_Student" will be a subclass of two classes, each coming from a different ontology. However, a given class can be subsumed by a single super-class that encompasses the union of two or more classes. So, "PhD_Student" can also be subsumed by a class that is the union of "Student" and "Researcher":

$$
\mathrm{O}_{3}=\{\text { PhD_Student } \sqsubseteq(\text { Student } \cup \text { Researcher })\}
$$

\section{Relational Redundancy}

Redundant information in an ontology is any explicit information that can already be inferred by an ontology reasoner. Redundant relations are implicitly mentioned in the ontology and useless to repeat [76]. In short, redundant relations or edges are those that are exactly repeated or those that can be deduced by other paths (i.e., by another sequence of relations). Unexpected relation redundancies may appear in an integrated ontology, especially the redundancy of subsumption relations. This is caused by the full merge of entities or by the addition of equivalence relations linking different entities in an integrated ontology. Equivalence relations are actually shortcuts for pairs of subsumption relations. Indeed, an equivalence relation between two entities (i.e., an equivalentClass or equivalentProperty axiom) is implicitly equal to two subsumption relations (i.e., two subClass Of or subPropertyOf axioms) in both directions, as stated in equation 11 where $e_{1}$ and $e_{2}$ are two entities (i.e., two concepts/classes or two object/datatype properties).

$$
\left\langle e_{1}, e_{2}, \equiv\right\rangle=\left\langle e_{1}, e_{2}, \sqsubseteq\right\rangle+\left\langle e_{2}, e_{1}, \sqsubseteq\right\rangle
$$

The redundancy of relations occurs when a merged concept subsumes or is subsumed by a source concept and a target concept that are linked by a subsumption or equivalence relation [77]. It also occurs when a merged individual is instantiated by a source concept and a target concept that are linked by a subsumption or equivalence relation [77]. There are many other redundancy cases as in the examples below:

Example 5 (Relation Redundancy). Consider the following two ontologies $O_{1}$ and $O_{2}$. In $O_{1}, A$ is a parent class of $B$. While in $\mathrm{O}_{2}, \mathrm{C}$ is a parent class of $D$. Formally,

$$
\begin{gathered}
O_{1}=\{B \sqsubseteq A\} \quad O_{2}=\{D \sqsubseteq C\} \\
\mathcal{A}=\left\{c_{1}, c_{2}\right\} \quad c_{1}=\left\langle A \sqsubseteq D>\quad c_{2}=<B \sqsubseteq C>\right.
\end{gathered}
$$

If the ontology matching step generates two correspondences $c_{1}$ and $c_{2}$ stating that $A$ is a subclass of $D$, and $B$ is a subclass of $C$, then the integrated ontology $O_{3}$ will yield a redundancy in the subsumption relations. The subsumption between $B$ and $C$ is redundant because it is already expressed by the three consecutive subsumptions between $B$ and $A$; $A$ and $D$; and $D$ and $C$.

Example 6 (Relation Redundancy). Consider another example also inspired by [69]. In $O_{1}$, we have two disjoint classes, "Employee" and "Student". In $\mathrm{O}_{2}$, we have not only "Employee" and "Student" classes which are not disjoint, but also "PhD_Researcher" and "Non_PhD_Researcher" classes which are sub-classes of "Student", such that "Employee" is disjoint with "Non_PhD_Researcher". Formally,

$$
O_{1}=\{\text { Employee } \perp \text { Student }\}
$$

$\mathrm{O}_{2}=\{$ PhD_Researcher $\sqsubseteq S$ tudent, Non_PhD_Researcher $\sqsubseteq$ Student, Employee $\perp$ Non_PhD_Researcher $\}$

In the fully merged ontology $O_{3}$, the disjointness between "Non_PhD_Researcher" and "Employee" (originating from $\mathrm{O}_{2}$ ) is already expressed by the disjointness between "Employee" and "Student" (originating from $\mathrm{O}_{1}$ ).

\subsubsection{Property's Domain and Range Oneness}

The principle of property's domain and range oneness [66, 78] (or the prohibition of property's domain and range multiplicity) states that object and datatype properties should have only one domain and only one range in the integrated ontology. When it comes to the range of datatype properties, this principle is specifically referred to as the principle of one datatype restriction [66]. The latter states that datatype properties should have only one range (i.e., only one datatype) in the integrated ontology. Usually, when we assign multiple domains/ranges to a property, we implicitly want to say that the property has a union of domains/ranges (i.e., has a domain/range that is composed of a disjunction of 
classes (or datatypes)). However, multiplicity has a totally opposite meaning when interpreted in a logical way. Indeed, although the OWL language allows a property to have multiple domains and ranges, it interprets this multiplicity as an intersection of domains or ranges (i.e., as a conjunction of classes or datatypes). Therefore, the ontology integration process should not introduce properties having multiple domains and/or ranges. Properties should rather have only one domain/range that composes the multiple domains/ranges using the union expression $<$ owl:unionOf $>$ [24].

It should be noted that domain and range statements are not constraints to be checked [79]. They are inference axioms (i.e., used by the ontology reasoner to infer further knowledge). In fact, domain and range statements are global restrictions (i.e., that have a global scope [80]). They do not behave as property restrictions that just restrict the property when it is associated with a particular class; they do restrict the property globally. For example, if we have a property $P$ having a domain $D$ and a range $R$ (i.e., $<P, r d f$ s:domain, $D>$ and $<P$, rdfs:range, $R>$ ), individuals $a$ and $b$, and a property assertion $\langle a, P, b\rangle$, then the reasoner will infer that $a$ is an instance of $D(<a, r d f$ :type, $D>)$ and that $b$ is an instance of $R(<b$ rdf:type, $R>)$ [81]. A domain or range violation happens when $a($ or $b)$ is not an instance of $D$ (or $R$ ). However, violating a domain or range constraint does not necessarily lead to an ontology inconsistency, but it can cause unexpected inferences that can lead to errors [79]. An ontology inconsistency will only be generated by a reasoner if the domain/range is constituted by multiple classes (or datatypes) that are disjoint with each other [81]. In this case, no individual (or no data literal) can be an instance of these disjoint domains or ranges.

Example 7 (Domain and Range Multiplicity). Consider the two ontologies $\mathrm{O}_{1}$ and $\mathrm{O}_{2}$. Both ontologies have the object property "studiesIn". In $O_{1}$, "studiesIn" has the class "Person" as a domain and the class "School" as a range. In $\mathrm{O}_{2}$, "studiesIn" has the class "Student" as a domain and the class "University" as a range. Formally,

$$
\begin{aligned}
& O_{1}=\{\text { Person } \stackrel{\text { domain }}{\longleftarrow} \text { studiesIn } \stackrel{\text { range }}{\longrightarrow} S \text { chool }\} \\
& \mathrm{O}_{2}=\{\text { S tudent } \stackrel{\text { domain }}{\longleftarrow} \text { studiesIn } \stackrel{\text { range }}{\longrightarrow} \text { University }\} \\
& \mathrm{O}_{3}=\{\text { Person, Student } \stackrel{\text { domain }}{\longleftarrow} \text { studiesIn } \stackrel{\text { range }}{\longrightarrow} \text { School, Univer sity }\} \\
& \mathrm{O}_{3}=\{(\text { Person } \cap S \text { tudent }) \stackrel{\text { domain }}{\longleftarrow} \text { studiesIn } \stackrel{\text { range }}{\longrightarrow}(\text { S chool } \cap \text { Univer sity })\}
\end{aligned}
$$

In the fully merged ontology $\mathrm{O}_{3}$, the property "studiesIn" will be merged and will have the two domains "Person" and "Student", and the two ranges "School" and "University". An ontology reasoning process will interpret the domain of "studiesIn" as the intersection (conjunction) of the two classes "Person" and "Student", and the range of "studiesIn" as the intersection (conjunction) of the two classes "School" and "University". The following object property assertion declares that "Ines" (which is an instance of the class "Student") studies in "Tunis_El_Manar_University" (which is an instance of the class "University"):

$$
\mathrm{O}_{3}=\{<\text { Ines } \longrightarrow \text { studiesIn } \longrightarrow \text { Tunis_El_Manar_University }>\}
$$

If a reasoning process is performed on the integrated ontology $\mathrm{O}_{3}$, the reasoner will infer that "Ines" is an instance of "Person" and an instance of "Student". Similarly, the reasoner will infer that "Tunis_El_Manar_University" is an instance of "School" and an instance of "University". If the class "Person" was disjoint with the class "Student" or if the class "School" was disjoint with the class "University", the ontology $\mathrm{O}_{3}$ would be inconsistent, because "Ines" and "Tunis_El_Manar_University" would be instances of two disjoint classes, which is impossible. When the classes "Person" and "Student" are not disjoint and the classes "School" and "University" are not disjoint (which is our case), the reasoner will not find any inconsistency (unless it finds one in another part of the ontology due to these inferences). A correct integration process would assign to the property "studiesIn" a domain composed of the union (disjunction) of "Person" an "Student", and a range composed of the union (disjunction) of "School" and "University", as follows:

$$
\mathrm{O}_{3}=\{(\text { Person } \cup \mathrm{S} \text { tudent }) \stackrel{\text { domain }}{\longleftarrow} \text { studiesIn } \stackrel{\text { range }}{\longrightarrow}(\text { S chool } \cup \text { University })\}
$$

Example 8 (Datatype Restriction Multiplicity). Consider an example proposed by Babalou and König-Ries [66]. Suppose that we have two ontologies $\mathrm{O}_{1}$ and $\mathrm{O}_{2}$. Both ontologies have the datatype property "author_Id". In $\mathrm{O}_{1}$, "author_Id" has the class "Person" as a domain and the datatype "String" as a range. In $\mathrm{O}_{2}$, "author_Id" has the class "Person" as a domain and the datatype "Integer" as a range. Formally,

$$
\begin{aligned}
& O_{1}=\{\text { Person } \stackrel{\text { domain }}{\longleftarrow} \text { author_Id } \stackrel{\text { range }}{\longrightarrow} \text { String }\} \\
& \mathrm{O}_{2}=\{\text { Person } \stackrel{\text { domain }}{\longleftarrow} \text { author_Id } \stackrel{\text { range }}{\longrightarrow} \text { Integer }\} \\
& \mathrm{O}_{3}=\{\text { Person } \stackrel{\text { domain }}{\longleftarrow} \text { author_Id } \stackrel{\text { range }}{\longrightarrow} \text { String, Integer }\}
\end{aligned}
$$




$$
\mathrm{O}_{3}=\{\text { Person } \stackrel{\text { domain }}{\longleftarrow} \text { author_Id } \stackrel{\text { range }}{\longrightarrow}(\text { S tring } \cap \text { Integer })\}
$$

In the fully merged ontology $\mathrm{O}_{3}$, the property "author_Id" will be merged, and will have the domain "Person" and the two ranges "String" and "Integer". An ontology reasoning process will interpret the range of "author_Id" as the intersection (conjunction) of the two datatypes "String" and "Integer". The following datatype property assertion declares that "Ines" (which is an instance of the class "Student") has the author ID "author_1234" (which is a literal of the datatype "String"):

$$
\mathrm{O}_{3}=\{<\text { Ines } \longrightarrow \text { author_Id } \longrightarrow \text { "author_1234" }>\}
$$

If a reasoning process is performed on the integrated ontology $\mathrm{O}_{3}$, the reasoner will infer that "Ines" is an instance of "Person". Similarly, the reasoner will infer that "author_1234" is a value of the datatype "String" and a value of the datatype "Integer". This will lead to the inconsistency of $\mathrm{O}_{3}$ (which is a fatal error), because the literal "author_1234" is not a String and an Integer at the same time. If the range datatypes had an overlap (such as the datatypes "Int", "Integer", "unsignedInt", "nonNegativeInt", and "positiveInt"; or the datatypes "Literal" and "PlainLiteral", etc), the ontology $\mathrm{O}_{3}$ could avoid being inconsistent. A correct integration process would assign to the property "author_Id" a range composed of the union (disjunction) of "String" and "Integer", as follows:

$$
\mathrm{O}_{3}=\{\text { Person } \stackrel{\text { domain }}{\longleftarrow} \text { author_Id } \stackrel{\text { range }}{\longrightarrow}(\text { String } \cup \text { Integer })\}
$$

\subsubsection{Entity Connectivity}

The principle of entity connectivity [66, 78] or unconnected entity prohibition states that no entity (class or property) should be isolated or unconnected in a given ontology. In other words, it states that entities that originally have some connections in the input ontologies should preserve these connections and not become totally unconnected in the integrated ontology. An unconnected entity is an entity that has no relation to the rest of the ontology. More precisely, a class or a property becomes unconnected when it does not have any associated subsumption relations in its description [78], thus it does not have any named parents, children or siblings. This definition can be relaxed by considering an entity unconnected when it also does not have any associated non-taxonomic relations in its description [78], such as equivalence or disjointness relations for classes and properties, and inverseOf relations for object properties. The ontology integration process can make some entities unconnected, especially the asymmetric integration case. The latter allows to remove some relations from the source (non-priority) ontologies in order fulfill coherence and/or conservativity principles for the target (priority) ontology.

\subsection{Ontology Integration Repair Strategies}

To ensure the integration principles, we have noticed two repair/debugging perceptions widely used in state-of-theart ontology integration research works. Some authors choose to alter the alignments because they are considered to be incoherent and non-conservative with respect to the input ontologies, while the input ontologies are considered to be always sound and much more reliable than alignments. Other authors choose to alter the input ontologies because they consider that whenever the alignments contain all the correct correspondences and yet the integrated ontology still contains coherence and conservativity violations, then these violations are only caused by the incompatible models of the input ontologies which have conceptual differences of the same domain. Finally, others choose to alter both the input ontologies and the alignments because they consider that conflicts can be caused either by alignments or by ontologies. Actually, the target of the repair approach is case dependent. For example, in Multi-Agent Systems, each agent uses a private knowledge encoded as an OWL ontology that cannot be altered, which makes the only possible repair solution is to agree on a common alignment between these agents. It may be the case that one or all (or none) of the input ontologies should be immutable.

State-of-the-art approaches generally remove elements from the alignments and/or from the input ontologies. They may also rarely add elements to the input ontologies for the correction of the conservativity principle. All these strategies should satisfy the principle of minimal change, sometimes called minimality. Its purpose is to avoid removing or adding axioms as much as possible, based on the number of removed correspondences from the alignments, or the number of removed axioms from the input ontologies, or rarely based on the number of inserted axioms to the input ontologies.

\subsubsection{Alignment Improvement}

We distinguish two types of alignment improvement [17]: the alignment repair which can be performed in the ontology matching module or in a separate module; and the alignment disambiguation which can also be used in the alignment repair. 
Alignment Repair. Alignment repair or repairing, (a.k.a. alignment debugging, alignment diagnosis, mapping revision, mapping repair, or briefly repair), aims at resolving the violations of coherence and/or conservativity by reducing the alignments and preserving the input ontologies being integrated.

An alignment should comply with the coherence principle. A coherent alignment [51, 82] is an alignment that does not violate the coherence principle by not introducing unsatisfiable entities in the integrated ontology. During the ontology matching step, matching tools should exploit the hidden semantics of the input ontologies, especially the disjointness information, to generate coherent correspondences. Current alignment repair tools are mostly based on the satisfiability checking, such as ALCOMO [51, 61], LogMap [83, 84], AML [43, 85], YAM++ [86], ASMOV [87], and Lily [88] repair facilities. They compute justifications by searching for the set of axioms entailing each unsatisfiable entity in the integrated ontology, then remove the minimal set of correspondences involved in the justifications. This process is called the minimal diagnosis process [89]. On the one hand, ALCOMO makes a complete reasoning for the justification by computing all possible justifications for each unsatisfiable class. Justifications can be disjointness axioms, domain restriction axioms, range restriction axioms, and all types of axioms in $\mathcal{E} \mathcal{L}^{++}[90,91]$ which is a fragment and a lightweight version of DL. This makes ALCOMO a non-scalable alignment repair tool because using the complete reasoning in the repair process is very expensive and inefficient. However, ALCOMO lets the user choose between complete or incomplete reasoning. On the other hand, LogMap-Repair and AML-repair make an incomplete reasoning that mainly computes one justification for each unsatisfiable class, namely a disjointness-based justification. They eliminate the incoherent correspondences that are involved in conflicts caused by disjointness relations between classes of the input ontologies. To address the scalability problem, occurring whenever there is a large number of unsatisfiabilities, most of these approaches rely on approximate algorithms, and thus perform an approximate (d) alignment repair. Apart from filtering out the incoherence-causing correspondences, some approaches, such as LogMap, may also alter the alignment by changing some equivalence correspondences to subsumption correspondences-like does the second method of the alignment disambiguation technique in the next paragraph.

An alignment should also comply with the conservativity principle. A conservative alignment is an alignment that does not violate the conservativity principle. It does not introduce new semantic relations between entities of an input ontology, especially new subsumption relations, in the integrated ontology. Correspondences leading to such alterations should be discarded from the alignment. In general, state-of-the-art approaches such as [55, 70, 71] works capture the amount of structural change between two given ontologies using the notion of logical difference [92, 93] or deductive difference [55]. In particular, they often use the approximation of the deductive difference [44] that captures the amount of structural change between the classification hierarchies of two given ontologies. These techniques compare the subsumption entailments of the resulting integrated ontology with the subsumption entailments of an input ontology-before being integrated. The purpose is to detect the set of subsumption entailments (i.e., inferred axioms) that do hold in the integrated ontology but do not hold in the original input ontology. This difference reflects the new subsumption entailments that were introduced to the input ontology after being integrated.

The alignment repair will restore the coherence and/or the conservativity of the produced alignment, but will generate redundancy/duplication and ambiguity in the integrated ontology.

Alignment Disambiguation. It aims at disambiguating or reducing a many-to-many alignment to obtain a one-to-one alignment. It corresponds to a bipartite matching problem. There are two approaches for dealing with ambiguous equivalence correspondences. The choice of the appropriate approach is case dependent [42].

1. The first approach considers that among the ambiguous equivalence correspondences, there is only one correct correspondence that truly reflects a synonym or alternative entity, while the remaining ones rather reflect similar, related or overlapping terms that do not strictly denote equivalent entities [42]. The simplest method to resolve the disambiguation problem is to always keep the equivalence correspondence that has the highest confidence value, and remove the remaining ones [17]. This approach consists in filtering correspondences having the same source entity or the same target entity by only keeping a single correspondence having the highest confidence value (See Figure 8). It is a greedy approach [17] that can be viewed as a Stable Marriage/Matching problem, which assigns one object from a first set to one and only one object from a second set [94].

An alternative solution is based on the principle of locality, which assumes that entities semantically related to entities of a correct correspondence are likely to be matched to each other by correct correspondences too. Semantically related entities are hierarchy neighbors such as more general entities (i.e., parents/ancestors) and more specific entities (i.e., children/descendants). Low confidence in the neighborhood of a correspondence can 
reveal its incorrectness. For example, if entities $e_{1}$ in $O_{1}$ and $e_{2}$ in $O_{2}$ are correctly matched, then the neighbors of $e_{1}$ are likely to be matched to the neighbors of $e_{2}$. If the correspondences relating the neighbors of $e_{1}$ and $e_{2}$ have low confidence values, then the correspondence $<e_{1} \equiv e_{2}>$ may be incorrect. Therefore, for each ambiguous correspondence, this disambiguation method counts the confidence proportion of correspondences reachable by super-entities and/or sub-entities of the matched entities, and finally only keeps the correspondence that has the highest ratio [95].

2. If one of the two matched ontologies is more granular or general than the other one (meaning that the terms of one ontology are more granular or general than those of the other ontology), then the ambiguous equivalence correspondences are considered as subsumption correspondences [96]. Indeed, an entity in the general ontology is decomposed into several entities in the granular ontology. Therefore, the second method to tackle the disambiguation problem proposes to refine equivalence relations to subsumption relations through changing their

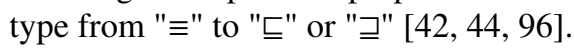

\subsubsection{Ontology Improvement}

We distinguish three types of ontology improvement: the ontology repair and the ontology correction, which are performed after the ontology matching module; and the ontology refinement, which is performed after the ontology merging module.

Ontology Repair (Ontology Axiom Exclusion). Ontology repair, a.k.a. ontology diagnosis, ontology debugging, or ontology revision, aims at fixing the violations of coherence and/or conservativity by reducing the input ontologies being integrated and preserving the produced alignment between them. Once a violation is detected, the strategy of axioms exclusion can be applied to solve it. It removes a minimal set of axioms (from the input ontologies) involved in logical conflicts and/or alterations in the integrated ontology, as do Babylon Health [42] and ContentMap [55]. Nevertheless, doing so leads to the loss of ontological knowledge that could sometimes be critical or of paramount importance for some applications or services.

Ontology Correction (Ontology Axiom Inclusion). Ontology correction aims at fixing the violations of conservativity by increasing the input ontologies being integrated and preserving the produced alignment between them. Once a conservativity violation is detected, the strategy of axioms inclusion can be applied to solve it. It adds to the input ontologies (each in isolation) a minimal set of axioms, such that they can entail novel axioms that were originally violating the conservativity principle in the integrated ontology [44, 97, 98].

Ontology Refinement or Pruning. Although structural redundancies, relational redundancies, subsumption cycles and unconnected entities do not semantically or logically affect the coherence of the integrated ontology, it is often necessary to refine or prune the integrated ontology to keep it understandable, sound and simple [77]. Refining an integrated ontology prunes its relational and structural redundancies, resolves its subsumption cycles, and removes (or connects) its unconnected entities, etc. To solve the cycles, Pottinger and Bernstein [65] came up with the idea of merging the cycle into a single entity and keeping all the properties of its combined entities. The intuition behind this is that the $i s-a$ ation is transitive; hence, a cycle of $i s$ - $a$ relations implies the equality of all its elements. As for the target-driven asymmetric merge, Raunich and Rahm [63,64] proposed to remove from the cycle one of the $i s-a$ relations that are originating from the source ontologies. Indeed, according to them, a full merge of two acyclic ontologies cannot involve only source relations or only target relations in a cycle. Similarly, to solve structural redundancies introduced in their integrated ontology, they proposed to remove the additional leaf paths that are originating from the source ontologies. Finally, to solve the unconnected concepts in their integrated ontology, Raunich and Rahm [63, 64] removed the source concepts that are no longer linked to any concept (via a subsumption relation).

Remark 1. Some authors (e.g., in [77, 78, 99]) consider the ontology repair (or ontology debugging) as one of the ontology refinement tasks. However, in this paper, we wanted to highlight two different ontology integration repair approaches, which are the alignment repair and the ontology repair. Therefore, we preferred to make a separation between ontology repair and ontology refinement. Indeed, we consider that ontology repair mainly resolves semantic/logical issues (such as ontology inconsistency and incoherence) which are serious errors in an ontology, whereas ontology refinement resolves additional issues that are much less serious because they do not involve semantics. 
We conclude that whenever ontologies describe conflicting domain perspectives, then blindly integrating them and ensuring both coherence (\& conservativity) and information preservation from ontologies and alignments is infeasible.

\section{Ontology Integration: Related Work}

In this section, we review the process of ontology integration, summarize the different integration methods used in the case of multiple input ontologies, then outline the most important research works on ontology integration, and finally put forth some initial observations.

\subsection{General Ontology Integration Process}

The general workflow of the ontology integration process covers the following main phases [100]:

1. Pre-processing Phase: It analyzes, evaluates and validates the selection of the input ontologies, normalizes them, and/or improves their quality, mainly whenever they originally contain conflicts or redundancies, in order to reduce the matching phase workload. For instance, if the ontologies to be matched are not expressed in the same language [101], then a normalization step is necessary to translate them from one language or formalism into a uniform representation (e.g., OWL) without changing their semantics;

2. Matching Phase: It identifies correspondences between the input ontologies - usually pairs of equivalent entities - and generates an alignment. It includes the following steps [32]: filtering/hashing, entity similarity comparison - using syntactic, structural and/or semantic matchers—, correspondences generation, and alignment repair (optional). A repairing step can be either performed during the matching step, or performed separately in a standalone step;

3. Merging Phase: It merges the selected input ontologies into an integrated ontology (See Subsection 4.2);

4. Post-processing Phase: It evaluates, repairs, and refines the resulting ontology by checking its consistency and coherence, resolving its cycles and its coherence $\&$ conservativity violations, and pruning its redundancies.

\subsection{Integration of Multiple Ontologies}

Most of the state-of-the-art research works have addressed the issue of integrating or merging only two input ontologies. In the case of more than two input ontologies, ontology integration or enrichment is carried out always in an incremental way, while ontology merging can be performed incrementally or non-incrementally. In the following, we introduce the notions of pairwise or binary ontology integration and holistic or $N$-ary ontology integration to respectively denote the incremental and non-incremental methods. Both approaches produce the same resulting integrated ontology.

\subsubsection{Pairwise Ontology Integration}

In the incremental ontology integration approach, an empty or initial target ontology $O^{*}$ is iteratively fed and extended with a set of source (or local) ontologies $O_{1}, O_{2}, \ldots, O_{n}$, as shown in Figure 2 For each iteration, the available pairwise ontology alignment $\mathcal{A}$ involving the two current ontologies being processed is used (e.g., in [102, 103]). When the number of input ontologies is equal to $N$, the integration process of these ontologies makes $N$ iterations (if we count the initialization step). In the example of Figure 2, the integration process goes through five iterations for integrating five ontologies:

1. $O^{*}=O_{1}$, then

2. $O^{*}=O_{12}=O_{1}+O_{2}+\mathcal{A}_{1-2}$, then

3. $O^{*}=O_{123}=O_{12}+O_{3}+\mathcal{A}_{12-3}$, then

4. $O^{*}=O_{1234}=O_{123}+O_{4}+\mathcal{A}_{123-4}$, then

5. $O^{*}=O_{12345}=O_{1234}+O_{5}+\mathcal{A}_{1234-5}$.

During the ontology integration process, $O^{*}$ is called an intermediate integrated ontology [78] and it gets larger after each iteration. At the end of the process, $O^{*}$ is called a final target (or global) integrated ontology. The post-processing (or refinement) step for $O^{*}$ can be performed progressively after each iteration, or at the end of the integration process. Normally, the order in which the source ontologies are included in the target ontology does not influence the resulting integrated target ontology. In the schema integration domain in general, the pairwise integration approach is called the ladder strategy [104]. This approach is not scalable because it becomes impractical when there is a large number of input ontologies. 


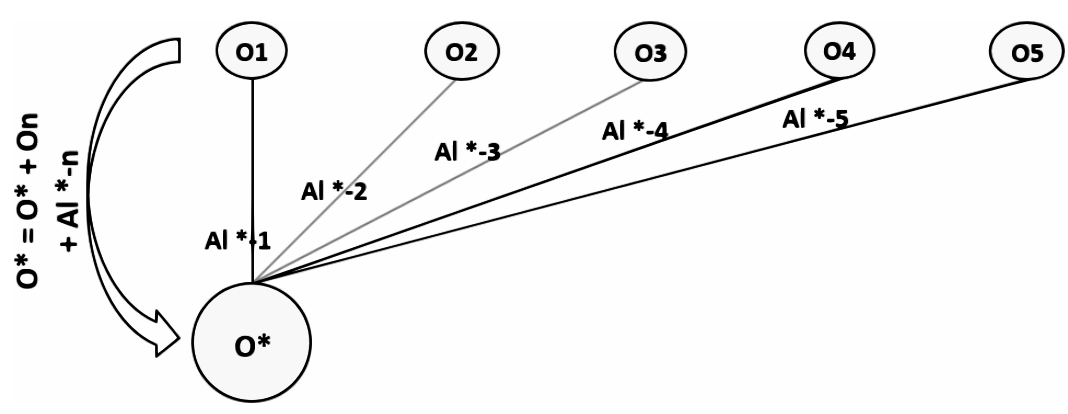

Figure 2: Pairwise Ontology Integration

\subsubsection{Holistic Ontology Integration}

In the non-incremental ontology integration approach, all the source (or local) ontologies $O_{1}, O_{2}, \ldots, O_{n}$ are combined together to constitute the target (or global) integrated ontology $O^{*}$. In this case, the whole process is carried out through a single iteration using the available holistic ontology alignment $\mathcal{A}$ involving all the source ontologies, as shown in Figure 3 . In the example of Figure 3 , the ontology integration process makes one iteration:

$$
\text { - } O^{*}=O_{12345}=O_{1}+O_{2}+O_{3}+O_{4}+O_{5}+\left[\mathcal{A}_{12345}\right]
$$

Otherwise, the whole process can be carried out in a single iteration using pairwise alignments between all ontology pairs, like in a normalized network of ontologies, as shown in Figure 4 (e.g., in [78, 99, 105, 106]). This method can be considered as the integration of a network of ontologies (See Subsection 3.6). In the schema integration domain in general, this method is called the balanced binary strategy [104]. In the example shown in Figure 4, the ontology integration process performs one iteration:

$$
\text { - } O^{*}=O_{12345}=O_{1}+O_{2}+O_{3}+O_{4}+O_{5}+\left[\mathcal{A}_{1-2}+\mathcal{A}_{1-3}+\mathcal{A}_{1-4}+\mathcal{A}_{1-5}+\mathcal{A}_{2-3}+\mathcal{A}_{2-4}+\mathcal{A}_{2-5}+\mathcal{A}_{3-4}+\mathcal{A}_{3-5}+\mathcal{A}_{4-5}\right]
$$

The holistic ontology integration approach is scalable because it is suitable for a large number of input ontologies.

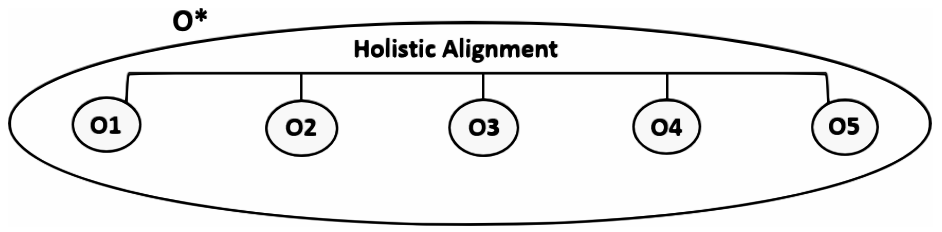

Figure 3: Holistic Ontology Integration



Figure 4: Holistic Ontology Integration using Pairwise Alignments

The pairwise ontology integration is much more adopted in the literature than the holistic ontology integration, where the latter remains a challenging task to perform. This is due to two reasons:

- First, the ontology matching community has always adopted the pairwise matching, because of its simple search space, and has rarely considered the holistic matching, which explains the unavailability of holistic ontology alignments; 
- Second, the current automated ontology matching systems have become quite proficient at generating pairwise ontology alignments, and particularly at identifying simple equivalence correspondences between two ontologies [32].

Remark 2. In some works, the integration is performed using only the pairwise alignments between the target ontology in its initial state and the source ontologies. They proceed in an incremental manner as shown in Figure 5a (e.g. in [42]) or in a non-incremental manner as shown in Figure $5 \mathrm{~b}$ (e.g. in [107]). These two processes do not really differ. In the example of Figure $5 \mathrm{a}$, the integration process makes five iterations:

1. $O^{*}=O_{1}$, then

2. $O^{*}=O_{12}=O_{1}+O_{2}+\mathcal{A}_{1-2}$, then

3. $O^{*}=O_{123}=O_{12}+O_{3}+\mathcal{A}_{1-3}$, then

4. $O^{*}=O_{1234}=O_{123}+O_{4}+\mathcal{A}_{1-4}$, then

5. $O^{*}=O_{12345}=O_{1234}+O_{5}+\mathcal{A}_{1-5}$.

Similarly, in Figure 5b, the ontology integration process makes one iteration:

- $O^{*}=O_{12345}=O_{1}+O_{2}+O_{3}+O_{4}+O_{5}+\left[\mathcal{A}_{1-2}+\mathcal{A}_{1-3}+\mathcal{A}_{1-4}+\mathcal{A}_{1-5}\right]$

Their downside is that they do not achieve a complete semantic interoperability between the ontologies that have been integrated. Indeed, source ontologies $\left(\mathrm{O}_{2}, \mathrm{O}_{3}, \mathrm{O}_{4}, \mathrm{O}_{5}\right)$ may share common entities that do not exist in the target ontology $\left(O_{1}\right)$. These common entities between the source ontologies will be redundant because they are not matched with each other. Thus, they are neither linked nor merged in the integrated ontology $\left(O^{*}\right)$.

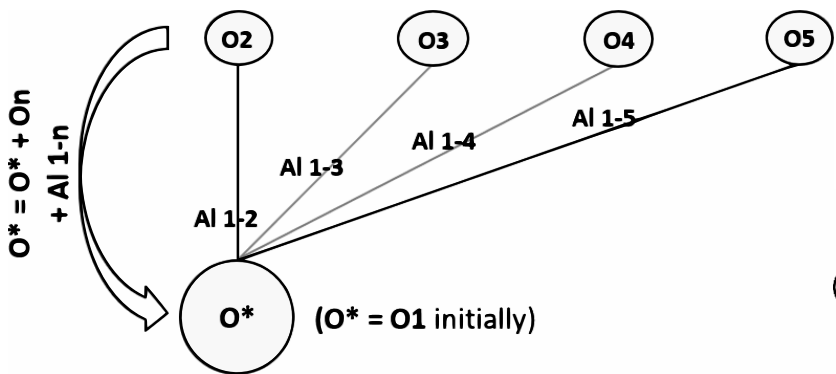

(a) Incremental/Pairwise Method

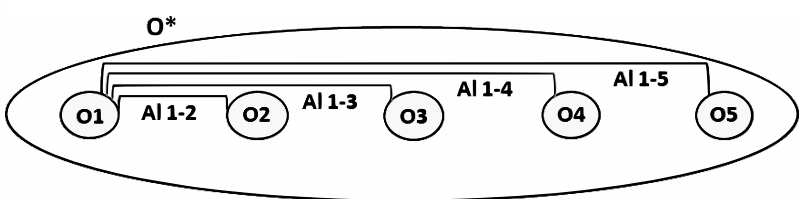

(b) Non-Incremental/Holistic Method

Figure 5: Ontology Integration Resulting in an Incomplete Semantic Interoperability

\subsection{Scrutiny of the Common Approaches}

In this subsection, we review the most relevant state-of-the-art research works related to the ontology integration. Research works that are only limited to the ontology matching or the ontology repair are beyond the scope of our paper.

Commonly, the ontology integration techniques can be assessed through the following criteria: the number of input ontologies to be integrated (i.e., two or more ontologies), the level of user involvement (i.e., automation or semiautomation), the information loss or incompleteness (i.e., preservation of the alignments and ontologies knowledge), the quality of the integrated ontology (i.e., violations, redundancies, cycles, etc), and the scalability. Table 3 sums up the most prominent approaches and their limitations. The mentioned works are listed in a chronological order. If a tool is semi-automatic or manual, then it cannot scale up, although achieving better results than do the automatic tools. Similarly, (coherence \& conservativity) repair and (ontologies \& alignments) information preservation can never be simultaneously achieved. 


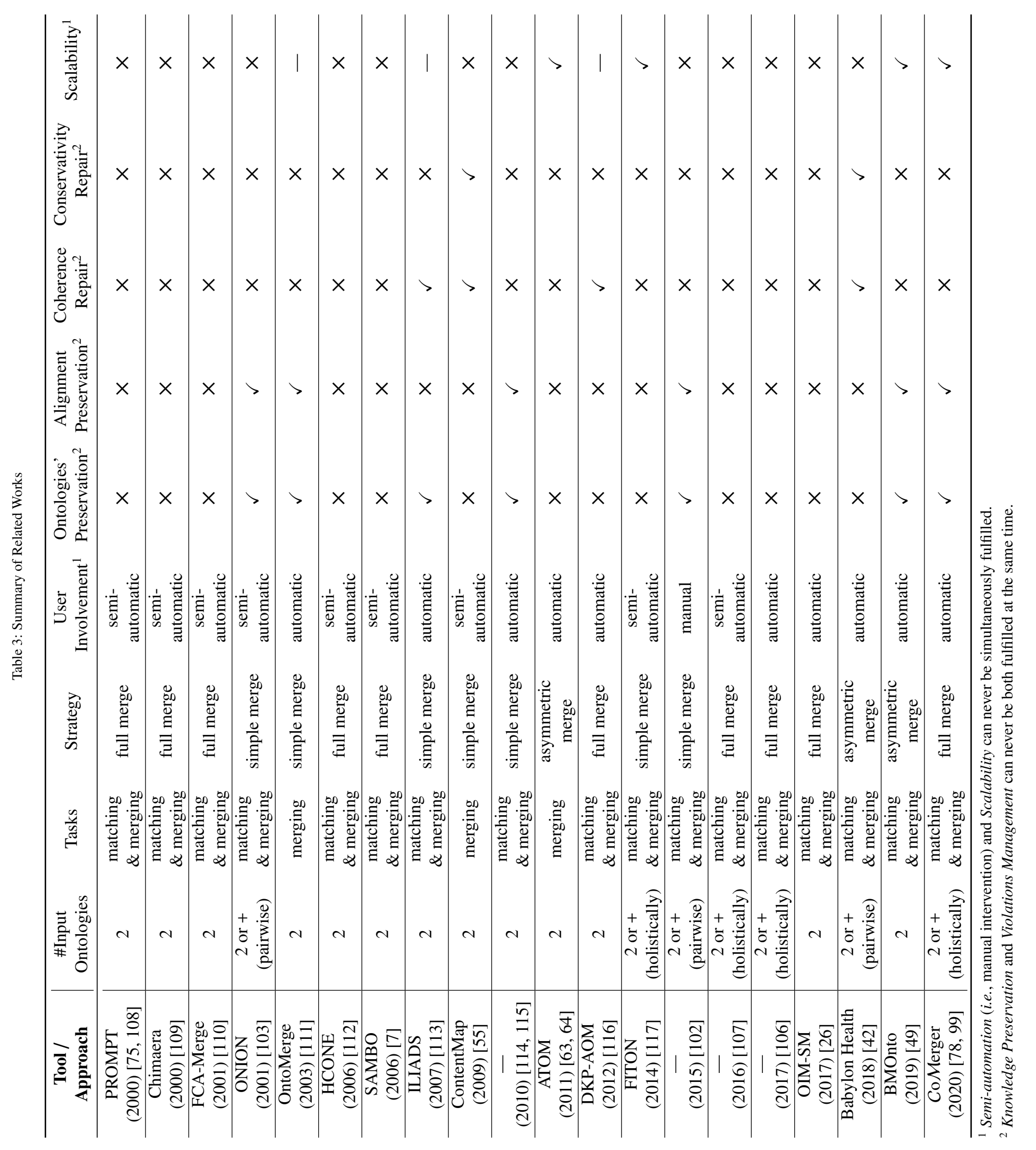




\subsection{Observations on the Related Work}

By taking a closer look at the related work, we make the following observations:

1. To avoid dealing with coherence violations in the integrated ontology, we notice that many integration and merging approaches do only preserve the hierarchy/taxonomy of the input ontologies (mainly the early research works) or do not particularly preserve the disjointness knowledge of the input ontologies (mainly the recent research works), e.g., [26, 27, 63, 64, 75, 106, 109], etc. Indeed, ontologies follow the Open World Assumption (OWA). The latter states that the concepts of an ontology are supposed to be shared and reused by multiple applications and users, because they are meant for an open distributed world such as the Web. Therefore, concepts are not disjoint by default—-they do overlap—and their description should be "closed off" where appropriate by disjointness relations. That is why, there are no unsatisfiable entities without disjointness axioms. It should be noted that coherence violations may also be caused by the use of disjointness correspondences, or by the use of implicit disjointness axioms not directly stated in the input ontologies. However, disjointness knowledge of the input ontologies can be of paramount importance in some applications, and removing it can sometimes be critical.

2. It is worth mentioning that many ontology integration works (mainly the early ones) do not evaluate their integrated ontology. They only focus on evaluating the quality of their ontology alignments resulting from the ontology matching step, e.g. in [7, 26, 106, 107, 112, 117].

3. We do also observe that most of the tools, in particular the early ones, are usually semi-automatic, requiring a lot of human intervention to either validate the suggested correspondences in the matching step, or to validate the actions to be performed in the merging step. This is especially the case when it comes to subsumption correspondences. Human intervention is also needed to resolve violations and redundancies either during or after the construction of the integrated ontology. Therefore, the result is usually dependent on the user observation or the expert decisions, e.g., in PROMPT [75, 108], Chimaera [109] and SAMBO [7], etc.

4. Finally, many research works are not generic. They are tailored to integrate only two ontologies that cover a predefined specific domain, since the integration of more than two ontologies at the same time is more complex.

By and large, the way in which the issue of ontology integration is handled has gradually evolved and matured over time. In the last decade, research works generally focused on providing a graphical interface for an interactive real-time visualization in the process of ontology matching and merging. In doing so, the user had the upper hand to accept or reject the results and visually compare entities of the input ontologies, e.g., in PROMPT [75, 108], Chimaera [109], $S A M B O$ [7] and iMERGE [118]. They only preserve the class hierarchy of the input ontologies and therefore only treat structural redundancies in the integrated ontology, e.g., in [26, 63, 64, 75, 109]. They also emphasize on the matching process by only evaluating the correctness and completeness of the alignment, and ignoring the evaluation of the integrated ontology, e.g., in [7, 26, 106, 107, 112,117]. In addition, they are generally able to integrate only two ontologies. However, nowadays, recent ontology integration approaches tend to be more generic, making it possible to jointly integrate more than two ontologies. Besides, they are moving towards full automation and scalability of their approaches. Unlike the previous works, recent ones try to preserve as much information as possible from both alignments and ontologies, and therefore treat all kinds of violations, redundancies, and cycles in the integrated ontology. They also focus on assessing the quality of the alignment as well as the integrated ontology.

\section{Ontology Integration: Evaluation Metrics}

Looking at the related work approaches from the previous section, we notice that there are various input ontologies, different ontology integration types, many input parameters, and diverse evaluation metrics being used in the studied papers. There is a clear tendency towards the use of Precision and Recall metrics in the early works for assessing the matching results (i.e., the alignments). However, recent works use other evaluation metrics for assessing the integrated ontology, e.g. number of unsatisfiable classes, logical difference, number of cycles, number of redundant entities, etc. This makes it hard to compare these ontology integration approaches and make general statements on their performance.

Evaluating ontology integration techniques is still an open issue. It is difficult to make a comparison between an integration result and a gold standard because there are no agreed quality metrics/measures for evaluating them, such as Precision, Recall, and F-Measure metrics in the Information Retrieval field. We suggest using Precision and Recall 
measures to compute false and missing axioms of a resulting integrated ontology compared to an ideal integrated ontology. However, there is a huge lack of references within the community. Besides, a perfect result is impossible to manually obtain for large ontologies, and there could be more than just one perfect result [27]. For the time being, there are no accepted benchmarks and gold standard criteria that can be used to objectively and generally assess the quality of the proposed integration approaches. There have been some attempts to produce benchmarks for ontology integration. The first existing benchmark [27] is not published and thus cannot be used, whereas the second one [119] is composed of only very small ontologies. Both proposed benchmarks result from an automatic asymmetric merge of two input ontologies. Inspired by [27, 42, 55, 64, 69, 78, 119] respective works, we gather the following metrics that can assess ontology integration results:

1. Entities coverage: Number of preserved entities from the input ontologies;
2. Axioms coverage/completeness: Number of preserved input ontologies' axioms;
3. Correspondences coverage: Number of preserved alignment correspondences;
4. Ontology consistency: Is the integrated ontology consistent? (True or False);
5. Ontology coherence: Number of unsatisfiable entities in the integrated ontology;
6. Logical difference of the input ontologies before and after being integrated;
7. Redundancy: Number of redundant/duplicated entities in the integrated ontology;
8. Ambiguity: Number of overlapping terms appearing in the entities of the ontology;
9. Leaf path difference of the input ontologies before and after being integrated;
10. Loops: Number of subsumption cycles in the integrated ontology;
11. Number of properties with multiple domains or ranges in the integrated ontology;
12. Number of unconnected entities (classes \& properties) in the integrated ontology;
13. Efficiency: The runtime performance;
14. Scalability: Scalable runtimes when using large and rich input ontologies;
15. Manual effort: Is the user involved in the ontology integration process?

The three first metrics are meant to evaluate the degree of information preservation or completeness. Coverage metrics for entities and axioms satisfy the principle of ontologies' knowledge preservation (See Subsection 5.1.2), while coverage of correspondences satisfies the principle of alignment preservation (See Subsection 5.1.3). They ensure that there is no information loss or incompleteness from the input ontologies and alignments. The metric of entities coverage [27, 64] can be split into classes coverage, object properties' coverage, data properties' coverage, and instances coverage. It reflects (the number or) the percentage of preserved entities in the resulting integrated ontology compared to the expected number of entities:

- For the simple merge case, the number of entities of the integrated ontology should be ideally equal to the sum of the entities of the input ontologies.

- For the full merge of two ontologies, the number of entities of the integrated ontology should be ideally equal to the sum of entities of the input ontologies, minus the number of merged entities (i.e., minus the number of equivalence ( $\equiv$ ) correspondences of the used non-ambiguous pairwise alignment).

- For the asymmetric merge case, the number of entities of the integrated ontology should be greater than the number of entities of the initial target ontology, and generally less than the sum of all entities of the input target and source ontologies.

The metric of axioms coverage [42] reflects the number (or the percentage) of preserved axioms in the integrated ontology. In addition to this metric, we can add two more specific metrics, that are subsumption axioms coverage and disjointness axioms coverage, to make sure that these particular types of axioms are preserved, since they are prone to be removed during the integration process. In the case of simple-merge and full-merge ontology integration, the metrics of entities and axioms coverage apply to all the input ontologies being integrated. That is, entities and axioms of the input ontologies should ideally be completely preserved. However, in the case of asymmetric-merge ontology integration, these (overall) metrics can be split into source coverage and target coverage [27]. Consequently, we can 
have six metrics: On the one hand, source entities coverage, target entities coverage, and (overall) entities coverage; On the other hand, source axioms coverage, target axioms coverage, and (overall) axioms coverage. The entities and axioms of the target ontology must be completely preserved. However, some entities and axioms of the source tologies can be missed. Both metrics of source entities and axioms coverage can be useful when comparing the source coverage of different ontology integration tools, in order to show the tool that most preserves the knowledge of the source ontologies. The metric of correspondences coverage [64] reflects the number (or the percentage) of preserved correspondences in the integrated ontology. When the used alignment also contains subsumption correspondences, the metric of correspondences coverage can be split into equivalence correspondences coverage $(=)$, "is- $a$ " correspondences coverage $(<)$, and "inverse is- $a$ " correspondences coverage $(>)$ [64].

Both of the fourth and fifth metrics satisfy the coherence principle (See Subsection 5.1.4). They assess the logical consistency of the integrated ontology and find the number of its unsatisfiable entities, mainly its unsatisfiable classes. The logical difference metric, denoted by $\operatorname{diff} \approx$ [44, 55] or $|\mathrm{LDiff}|$ [42], fulfills the conservativity principle (See Subsection 5.1.5). It finds, in the integrated ontology, the number of axioms that have been added to the original description of the input ontologies. In other words, it is the difference in the number of axioms of the input ontologies before and after being integrated. The two metrics of entities redundancy and entities ambiguity fulfill the minimality principle in the integrated ontology (See Subsection 5.1.6). They can be divided into classes redundancy and classes ambiguity, properties redundancy and properties ambiguity, and instances redundancy and instances ambiguity [66].

The number of loops (or is-a cycles) fulfills the acyclicity principle in the integrated ontology (See Subsection 5.1.7). We can divide this metric into class cycles and property cycles [66]. The metric of leaf path difference, denoted by $\Delta$ leaf paths [63, 64], satisfies the principle of structural redundancy prohibition in the integrated ontology (See Subsection 5.1.8). It is the difference in the number of leaf $i s$ - $a$-paths of the input ontologies before and after being integrated. This number reflects the amount of structural redundancies introduced after the integration. As for the principle of relational redundancy prohibition (See Subsection 5.1.8), to the best of our knowledge, there is no existing metric that can reflect the redundancy of relations or paths in an ontology. The number of (object and datatype) properties that have multiple domains or ranges satisfies the principle of property's domain and range oneness in the integrated ontology (See Subsection 5.1.9). The number of unconnected classes and properties in the integrated ontology satisfies the principle of entity connectivity (See Subsection 5.1.10, Metrics 5, 6, 7, 8, 9, 10, 11, and 12 should ideally be equal to 0 , but they do strongly depend on the quality of the input ontologies that have been integrated.

Efficiency and scalability are performance evaluation criteria. Efficiency means that the runtime of the ontology integration algorithm should compete with runtimes of the existing algorithms. As for the scalability criterion, the algorithm should be able to provide good performance and acceptable runtimes for large and heavyweight ontologies having hundreds of thousands of entities and axioms. Finally, manual effort is a user-related evaluation criterion. The user or expert intervention should be minimal, and it would be even better if the algorithm was fully automatic without any human intervention.

Although they belong to the ontology matching domain, Precision, Recall, and F-Measure can be included for evaluating the quality of the used alignment against a reference alignment. These three metrics are adopted by the Ontology Alignment Evaluation Initiative (OAEI) $\sqrt{6}$ campaign. They ensure the alignment correctness and completeness principle (See Subsection 5.1.1).

\section{Application Case for Future Investigation}

In this section, we focus on the integration of a network of ontologies, i.e. where we perform a holistic ontology integration using pairwise alignments (See Subsection 6.2.2). We aim to investigate the challenges of this particular ontology integration case, which illustrates the issues being faced, and then elaborate a set of learned lessons for the topic from the results of this experimentation.

\subsection{General Process of the Experimentation}

Nowadays, there are many available good-quality alignments resulting from well-known ontology matching systems. Leveraging them will help have trustworthy ontology integration results. We propose to holistically integrate two

\footnotetext{
${ }^{6}$ http://oaei.ontologymatching.org/
} 
or more independently developed ontologies, using reference alignments between all ontology pairs, as shown in Figure 4. To do so, we have developed two algorithms: OIAR (Ontology Integration with Alignment Reuse) and AROM (Alignments Reuse for Ontology Merging) to automatically build a simple-merge ontology and a full-merge ontology, respectively. Both of them have been developed using the OWL API [120], which is a Java API for developing, manipulating, and serializing OWL ontologies. OIAR and AROM take as input two or more OWL input ontologies to be integrated, one or more RDF alignments described in the Alignment API format [40], a new IRI or URI as a namespace for the future integrated ontology, and a confidence threshold ranging between $[0,1]$ to trim correspondences of the input alignment(s), i.e. to exclude correspondences below a given confidence/similarity value. They preserve all entities and axioms from the input ontologies and all correspondences from the input alignments (See Table 4). Source code and results of OIAR $\mathrm{D}^{7}$ and $\mathrm{AROM}^{8}$ can be downloaded from the GitHub platform.

Table 4: Comparison between OIAR and AROM

\begin{tabular}{|c|c|c|c|c|c|c|c|c|c|}
\hline Tool & $\begin{array}{c}\text { \#Input } \\
\text { Ontologies }\end{array}$ & Tasks & Strategy & $\begin{array}{c}\text { User } \\
\text { Involvement }\end{array}$ & $\begin{array}{l}\text { Ontologies' } \\
\text { Preservation }\end{array}$ & $\begin{array}{c}\text { Alignment } \\
\text { Preservation }\end{array}$ & $\begin{array}{l}\text { Coherence } \\
\text { Repair }\end{array}$ & $\begin{array}{l}\text { Conservativity } \\
\text { Repair }\end{array}$ & Scalability \\
\hline OIAR & $\begin{array}{c}2 \text { or }+ \\
\text { (holistically) }\end{array}$ & merging & simple merge & automatic & $\checkmark$ & $\checkmark$ & $x$ & $x$ & $\checkmark$ \\
\hline AROM & $\begin{array}{c}2 \text { or }+ \\
\text { (holistically) }\end{array}$ & merging & full merge & automatic & $\checkmark$ & $\checkmark$ & $x$ & $x$ & $\checkmark$ \\
\hline
\end{tabular}

We have chosen to carry out the experiments on the Large Biomedical Ontologies (LargeBio) track provided by the OAEI 2020 campaign. Ontologies, reference alignments and participant alignments are all downloadable from the OAEI website. LargeBio is composed of three large and semantically rich ontologies (See Table 5), namely FMA (Foundational Model of Anatomy), SNOMED-CT (Clinical Terms), and NCI (National Cancer Institute Thesaurus).

Table 5: Number of Entities in the LargeBio Ontologies

\begin{tabular}{l|rrrrr}
\hline LargeBio & Classes & Object Prop. & Data Prop. & Instances & Logical Axioms \\
\hline FMA & 78,988 & 0 & 54 & 0 & 79,218 \\
NCI & 66,724 & 123 & 67 & 0 & 96,046 \\
SNOMED-CT & 122,464 & 55 & 0 & 0 & 191,203 \\
\hline Total & 268,176 & 178 & 121 & 0 & 366,467 \\
\hline
\end{tabular}

Table 6: Number of Correspondences in the LargeBio Reference Alignments

\begin{tabular}{l|rrr|rrr}
\hline \multirow{2}{*}{ Alignment } & \multicolumn{3}{c|}{ Original } & \multicolumn{3}{c}{ Disambiguated } \\
\cline { 2 - 7 } & $\equiv$ & $?^{\star}$ & Total & $\equiv$ & $?^{\star}$ & Total $^{*}$ \\
\hline FMA-NCI & 2,686 & 338 & 3,024 & 2,369 & 190 & 2,559 \\
FMA-SNOMED & 6,026 & 2,982 & 9,008 & 5,209 & 2,579 & 7,788 \\
SNOMED-NCI & 17,210 & 1,634 & 18,844 & 13,606 & 790 & 14,396 \\
\hline Total Correspondences & $25,922^{* \dagger}$ & 4,954 & $30,876^{\dagger}$ & $21,184^{* *}$ & 3,559 & $24,743^{*}$ \\
\hline
\end{tabular}

* When these incoherence-causing correspondences are deleted, the alignment becomes repaired.

The original reference alignments contain 30,876 correspondences.

${ }^{\dagger \dagger}$ The repaired reference alignments contain 25, 922 correspondences.

The disambiguated reference alignments contain 24,743 correspondences.

The disambiguated $\mathcal{F}$ repaired reference alignments contain 21,184 correspondences.

The OAEI benchmark provides reference alignments between each pair of the LargeBio ontologies based on the UMLS meta-thesaurus [121] (See Table 6). In the reference alignments, correspondences having relations flagged by

${ }^{7}$ https://github.com/inesosman/OIAR

$\varepsilon^{8}$ https://github.com/inesosman/AROM 
"?" are correct equivalence correspondences involved in coherence violations detected by ALCOMO, LogMap, and/or $A M L$ alignment repair facilities. As a result, LargeBio reference alignments are only repaired for coherence violations, not also for conservativity violations.

We have integrated the three LargeBio ontologies using their three pairwise reference alignments—between each pair of them. The IRI of our integrated output ontology is http://integration. In the remainder, we omit IRI/URI prefixes of the entities for readability reasons. All tests have been performed with a confidence threshold equal to 0.0 , thus we have kept all the correspondences of the input alignments. It should be recalled that the higher the input confidence threshold, the lower the number of unsatisfiable entities in the integrated ontology. In addition, integrating ontologies from different domains always generates fewer unsatisfiable classes than integrating ontologies from the same domain. In the following, we explain the reasons for adding an alignment disambiguation module in some of our tests.

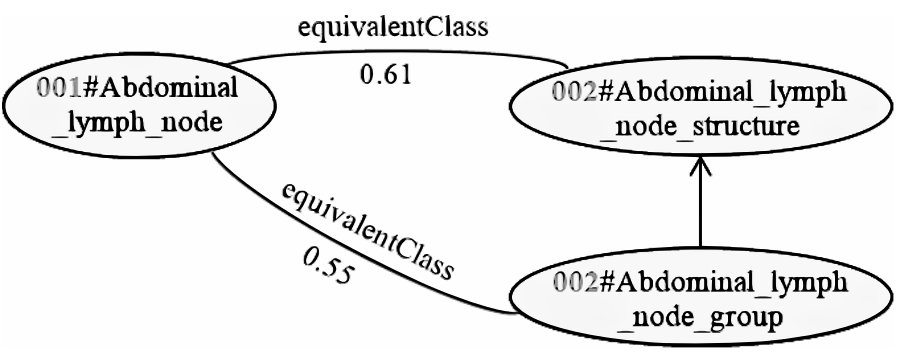

(a) in a Simple-Merge Ontology

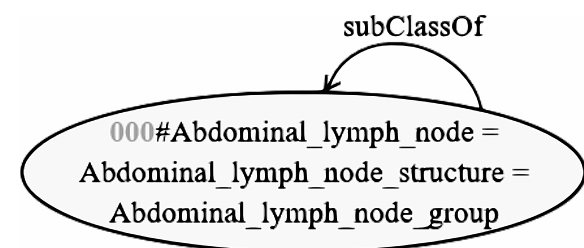

(b) in a Full-Merge Ontology

Figure 6: Two Ambiguous Equivalence Correspondences Leading to Redundancies and Cycles

Example 9. Figure 6 illustrates a snippet from the integration of FMA $\left(\mathrm{O}_{1}\right)$ and SNOMED-CT $\left(\mathrm{O}_{2}\right)$. It shows subsumption redundancies and a subsumption cycle formed because of the addition of two equivalence correspondences having the same source class "001\#Abdominal_lymph_node". In fact, in the simple-merge ontology (Figure 6a), each equivalence axiom linking two classes is formally equivalent to two subsumption axioms in both directions. However, in the full-merge ontology (Figure 6b), the three equivalent classes are merged together to constitute a single class that becomes subsumed by itself, since the subsumption between "002\#Abdominal_lymph_node_group" and "002\#Abdominal_lymph_node_structure" classes is conserved. This conserved subsumption is redundant because any class is subsumed by itself by default.

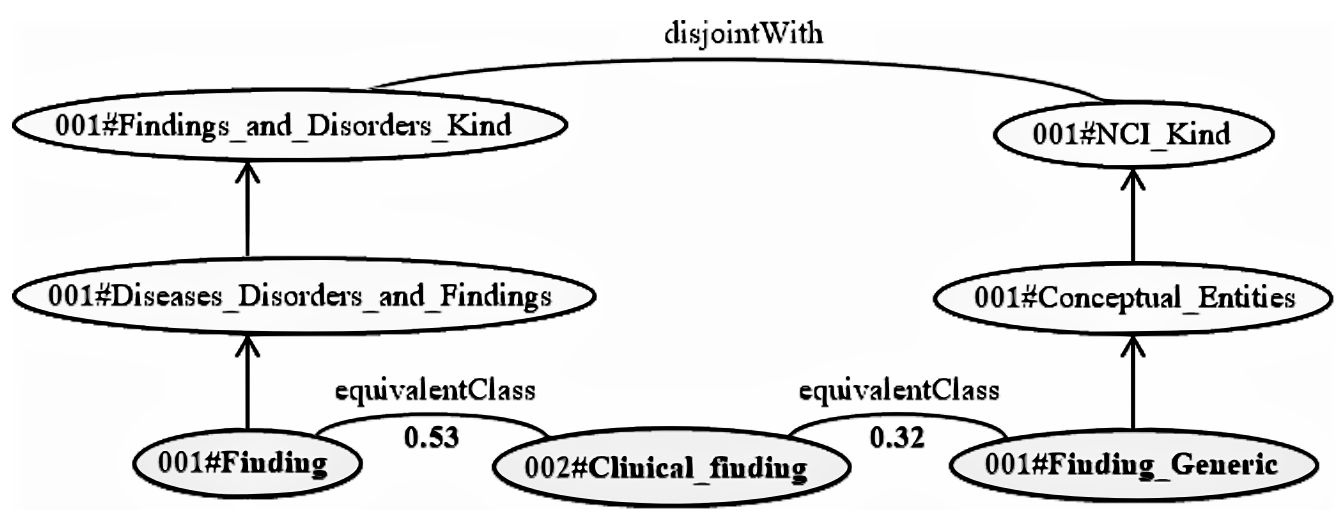

Figure 7: Two Ambiguous Equivalence Correspondences Leading to Unsatisfiable Classes in a Simple-Merge Ontology (or Leading to an Unsatisfiable Class in a Full-Merge Ontology)

Example 10. Figure 7 depicts a snippet from the integration of $N C I\left(\mathrm{O}_{1}\right)$ and SNOMED-CT $\left(\mathrm{O}_{2}\right)$. It represents unsatisfiable classes in dark grey, formed by the addition of two ambiguous equivalence correspondences having the 
same source class "002\#Clinical_finding". The three classes "001\#Finding", "002\#Clinical_finding" and "001\#Finding_Generic" become, by inference, sub-classes of the two disjoint classes "001\#Findings_and_Disorders_Kind" and "O01\#NCI_Kind" where the disjointness information comes from $\mathrm{NCI}\left(\mathrm{O}_{1}\right)$. This contradiction makes the three classes unsatisfiable.

In this case, if the DisjointWith axiom is removed, then we will avoid all these unsatisfiable classes. Nevertheless, the structure/hierarchy of the first input ontology (NCI) will be altered, and thus the conservativity principle will be violated. Indeed, the class "001\#Finding_Generic" will be a subclass of the class "001\#Finding" and all its parents, and the class "001\#Finding" will be a subclass of the class "001\#Finding_Generic" and all its parents, which was not originally stated in this ontology at first. However, if the class "002\#Clinical_finding", that belongs to the second input ontology (SNOMED-CT), is matched to only one class from the other ontology, precisely to the class "O01\#Finding" with which it has the highest similarity value, then we will avoid all these unsatisfiable classes, conserve the disjointness axiom, and satisfy the conservativity principle. Therefore, the alignment repair process would remove the correspondence between "002\#Clinical_finding" and "001\#Finding_Generic".

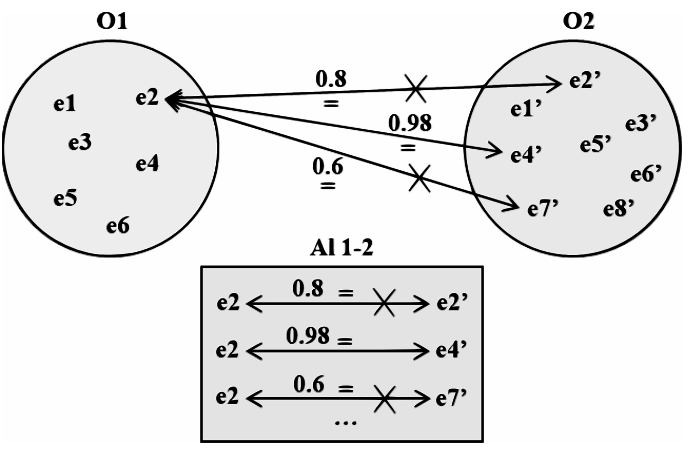

(a) Step 1

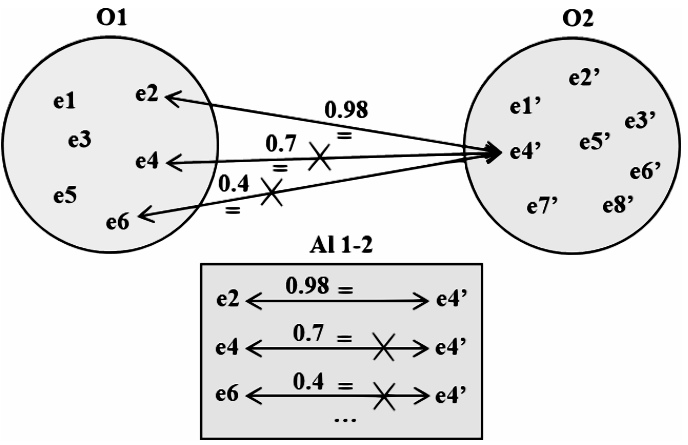

(b) Step 2

Figure 8: Ontology Alignment Disambiguation (Approach 1

For this reason, in some tests, we disambiguate the ambiguous equivalence correspondences. The disambiguation step is explained in subsection 5.2.1 and depicted in Figure 8 In this disambiguation, we filter out correspondences having the same source entity (as shown in Figure 8a), then we filter out correspondences having the same target entity (as shown in Figure $8 \mathrm{~b}$ by only keeping the most confident correspondence that has the highest value. If it happens that all ambiguous correspondences have exactly the same confidence value, we have decided to keep all of them, because if we randomly choose one of them, then the results will differ for each chosen correspondence.

\subsection{Experimentation Results}

Tables 7 and 8 sketch the quality of the ontologies resulting from integrating LargeBio ontologies using OIAR and AROM respectively, such that all axioms of the input ontologies are preserved. The Original column means that we have kept the input alignments ambiguous and unrepaired (i.e., we have used all existing correspondences from the input alignments). The Disambiguated column means that we have disambiguated the input alignments by only keeping one correspondence from each set of ambiguous correspondences (i.e., we have used all correspondences from the disambiguated input alignments). The Repaired column means that we have repaired the input alignments by removing their correspondences that have a relation "?" (i.e., we have only used correspondences having relations "I" from the input alignments). The Disambiguated E Repaired column means that we have disambiguated and repaired the input alignments (i.e., we have only used correspondences having relations " $\equiv "$ from the disambiguated input alignments).

Example 11. After the integration of FMA $\left(\mathrm{O}_{1}\right), \mathrm{NCI}\left(\mathrm{O}_{2}\right)$ and SNOMED-CT $\left(\mathrm{O}_{3}\right)$, this is an example of the unsatisfiable classes that were introduced in our resulting integrated ontology despite the repair and the disambiguation of the input reference alignments. Figure 9 shows two unsatisfiable classes: "001\#Portion_of_cytosol" from FMA and "O02\#Cytoplasmic_Matrix" from NCI. After adding two non-ambiguous equivalence correspondences, they become, by 
inference, sub-classes of the two disjoint classes "001\#Portion_of_body_substance" and "001\#Anatomical_structure", where the disjointness information comes from FMA. Here, to ensure the coherence of the integrated ontology, we will face a dilemma between sacrificing an equivalence correspondence from the alignment, which will reduce interoperability between ontologies, or sacrificing the disjointness axiom from the input ontology FMA, which will be a knowledge loss.

It is important to mention that when we integrate the three LargeBio ontologies using all correspondences from the original (unrepaired and ambiguous) reference alignments and without conserving any DisjointWith axiom from the input ontologies, we do not get any unsatisfiable class in our integrated ontology. In this case, our integrated ontology is consistent and coherent, but incomplete, i.e., lacking valuable disjoint knowledge. This proves that disjointness axioms are the main cause of semantic conflicts in the integration of LargeBio ontologies. It should also be noted that the full merge ontology always generates fewer unsatisfiable entities than does the simple merge ontology because it naturally contains fewer entities after they have been fully merged. Nevertheless, performing a full merge or a simple merge is exactly the same from a semantic point of view. In other terms, if one leads to unsatisfiable entities or an inconsistency, then the other will do so; and if one does not lead to unsatisfiable entities or an inconsistency, then the other will do so.

\subsection{Takeaway Lessons from the Experimentation}

In the following, we draw the attention of the reader to two lessons that we can figure out from our experimentation results:

Lesson 1: When we get all these unsatisfiable classes in the integrated ontology, we can doubt the correctness of the OAEI reference correspondences. OAEI reference alignments are directly extracted from the UMLS metathesaurus, which is the most comprehensive effort for integrating independently developed medical terminologies and ontologies. Pesquita $e t$ al. [123] proved that the original unrepaired reference alignments of the OAEI LargeBio track do contain erroneous correspondences [124], and that $A L C O M O, \log M a p$ and $A M L$ repair facilities, used by LargeBio reference alignments, do sometimes remove or alter correct correspondences. Overall, LargeBio reference alignments do contain some erroneous or missing correspondences, but even if they were totally perfect, we still cannot escape unsatisfiabilities. In fact, in a network of ontologies, incoherence can come either from a local incoherence in a particular ontology or alignment, or from a global incoherence between them [105]. However, in the case here, we believe that these unsatisfiable classes are beyond the abilities of the common alignment repair systems. It is worth noting that the current alignment repair systems do not deal with the simultaneous integration of multiple ontologies, and are dedicated to only integrating two ontologies using an alignment between them. Indeed, if we integrate two LargeBio ontologies using a repaired reference alignment between them, then we obtain a consistent and coherent ontology, i.e. that has no unsatisfiable classes. However, if we integrate more than two ontologies using several repaired pairwise alignments (between ontology pairs), we obtain an ontology that can have considerable unsatisfiable classes. This proves the compelling need for alignment repair systems that would be able to deal with networks of ontologies. In [105], Euzenat pointed out new perspectives on this challenging issue worth of exploration, namely, repairing networks of ontologies.

Lesson 2: Let us compare the two columns Disambiguated alignments and Repaired alignments from Tables 7 and 8 We observe that although the alignment disambiguation removes more correspondences than does the alignment repair, the use of the disambiguated alignments generates much more unsatisfiable classes than the use of the repaired alignments. We deduce that the alignment disambiguation is an "aggressive" approach that removes unnecessary correspondences without being able to guarantee coherence in the integrated ontology. It should be noted that the repaired alignments do contain ambiguous correspondences (as shown in Table 6). Despite being ambiguous, LargeBio repaired reference alignments do not lead to any unsatisfiable classes when we use them to integrate each LargeBio ontology pair separately. In other words, when we integrate a LargeBio ontology pair using its repaired reference alignment, we do not get any unsatisfiable classes in the integrated ontology. In this case, disambiguating the repaired alignment is useless and will generate many more redundant classes in the integrated ontology. To sum up, it is important to know that not all ambiguous equivalence correspondences generate unsatisfiable entities in the integrated ontology. However, alignment repair approaches may include an alignment disambiguation step in some cases, whenever needed. 


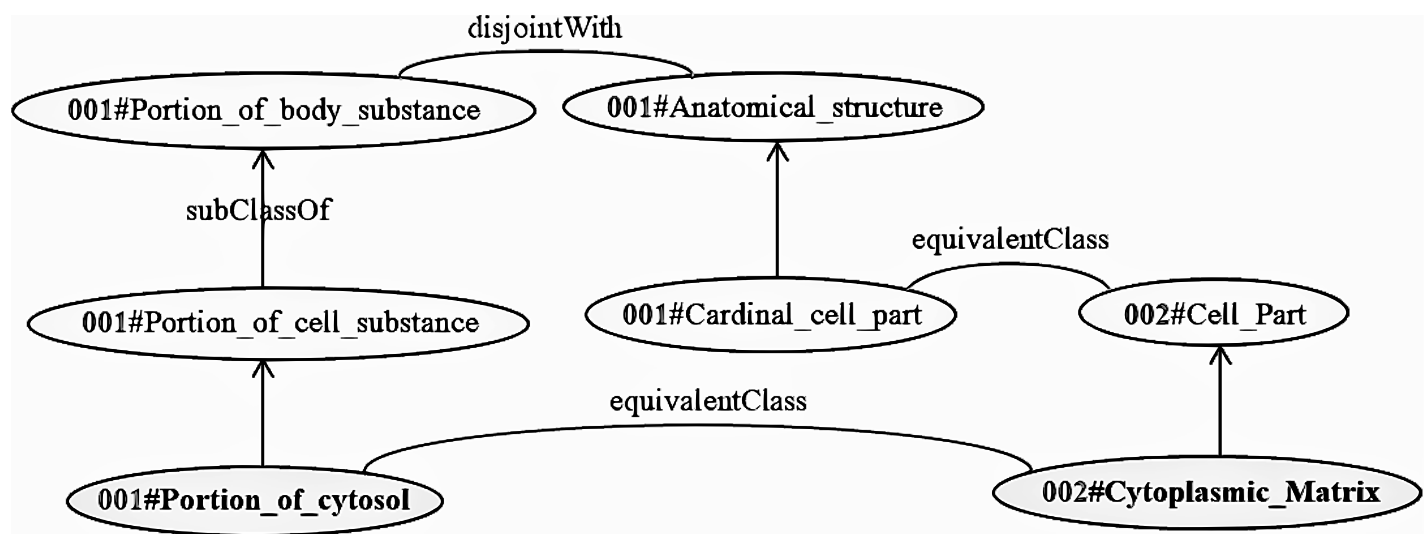

Figure 9: Unsatisfiable Class Formation in a Simple-Merge Ontology

Table 7: Quality of the Ontology Resulting from the Integration of LargeBio Ontologies using OIAR

\begin{tabular}{|c|c|c|c|c|}
\hline \multirow{2}{*}{$\begin{array}{c}\text { Simple-Merge } \\
\text { Integrated Ontology } \\
\text { Features }\end{array}$} & \multicolumn{4}{|c|}{ Input Alignments } \\
\hline & Original & Repaired & Disambiguated & $\begin{array}{l}\text { Disambiguated } \\
\text { \& Repaired }\end{array}$ \\
\hline $\begin{array}{l}\text { Classes } \\
\text { Object Properties } \\
\text { Datatype Properties } \\
\text { Instances }\end{array}$ & & & $\begin{array}{c}268,176 \\
178 \\
121 \\
0\end{array}$ & \\
\hline Logical Axioms ${ }^{1}$ & $\begin{array}{r}397,343 \\
(366,467 \\
+30,876)\end{array}$ & $\begin{array}{r}392,389 \\
(366,467 \\
+25,922)\end{array}$ & $\begin{array}{r}391,210 \\
(366,467 \\
+24,743)\end{array}$ & $\begin{array}{r}387,651 \\
(366,467 \\
+21,184)\end{array}$ \\
\hline Consistency $^{2}$ & $\checkmark$ & $\checkmark$ & $\checkmark$ & $\checkmark$ \\
\hline Unsatisfiable Classes $^{2}$ & 203,675 & 49,046 & 155,775 & 43,078 \\
\hline Redundant Classes & 0 & 8,498 & 4,501 & 10,540 \\
\hline Runtime $^{3}(\min )$ & 0.70 & 0.68 & 0.71 & 0.70 \\
\hline
\end{tabular}

${ }^{1}$ In parentheses, 366,467 is the total number of axioms of all input ontologies, and the value after the sum operator is the total number of correspondences of all input alignments.

${ }^{2}$ Computed using ELK, which is one of the fastest $\mathcal{E} \mathcal{L}$ reasoners for large ontologies [122].

${ }^{3}$ Runtimes do not include matching times since we take pre-established alignments as input.

Table 8: Quality of the Ontology Resulting from the Integration of LargeBio Ontologies using AROM

\begin{tabular}{l|rrrr}
\hline \multicolumn{1}{c}{ Full-Merge } & \multicolumn{4}{c}{ Input Alignments } \\
\cline { 2 - 5 } $\begin{array}{c}\text { Integrated Ontology } \\
\text { Features }\end{array}$ & Original & Repaired & Disambiguated & $\begin{array}{c}\text { Disambiguated } \\
\text { \& Repaired }\end{array}$ \\
\hline Classes & 240,634 & 244,173 & 245,334 & 248,097 \\
Object Properties & & & 178 & \\
Datatype Properties & & & 121 & \\
Instances & & & 0 & 363,135 \\
Logical Axioms & 359,600 & 360,577 & 362,404 & $\checkmark$ \\
Consistency & $\checkmark$ & $\checkmark$ & $\checkmark$ & 38,067 \\
Unsatisfiable Classes $^{1}$ & 177,975 & 42,450 & 138,523 & 10,540 \\
Redundant Classes $^{\text {Runtime }}{ }^{2}$ (min) & 0 & 8,498 & 4,501 & 0.77 \\
\hline
\end{tabular}

${ }^{1}$ Computed using the ELK ontology reasoner [122].

${ }^{2}$ Runtimes do not include matching times and alignment repair times. 


\section{Findings from the Survey} the evaluation of the integrated ontology.

\subsection{Ontology Integration Repair}

If the integration goal is interoperating different applications, then we believe that we should be strict with respect to the preservation of the input ontologies. Thus, no axiom from the input ontologies is allowed to be removed, and we should rather sacrifice correspondences by only repairing alignments, although this will decrease the semantic interoperability of applications. However, if the integration goal is to create a new ontology for a particular purpose, then we believe that the process can be relaxed with respect to the preservation of the input ontologies. Thus, removing axioms from the input ontologies can be tolerated, and the fulfillment of the conservativity principle is no longer mandatory. Consequently, we can preserve all correspondences from the original alignment in order to avoid the redundancy and ambiguity of entities in the resulting integrated ontology.

Since simple- and full- merge will always be torn between completeness, coherence (and also conservativity) such that we can never obtain a perfect result, the asymmetric merge or enrichment turns out to be the most successful integration type. At least, it surely achieves completeness and conservativity for the target seed ontology, and it surely achieves coherence for the resulting target integrated ontology. We think that it is particularly well suited to the integration case that aims to build an application-specific ontology. To sum up, integrating ontologies with incompatible/conflicting domain views or models is still an open and thriving issue [123]. However, if the asymmetric merge (enrichment) is acceptable in some ontology integration scenarios, then it can be a possible solution to this issue.

When dealing with violations, we can observe that most state-of-the-art repair approaches often choose the alignment repair over the ontology repair. Ontology integration works prefer repairing ontology alignments because there are already many ontology matching tools that perform with alignment repair, contrary to ontology repair that is much less experimented in the ontology integration area. However, many ontology integration scenarios actually suit to a more balanced repair approach between the input alignments and the input ontologies. The most common limitations in alignment repair works are scalability and complete reasoning. Besides, if there are no disjointness axioms declared in the input ontologies, then this may badly affect the quality of the alignment repair results.

In the reported ontology integration literature, we can underscore that the conservativity principle is much less investigated than the coherence principle. Indeed, tools that automatically detect and repair correspondences leading to a logical incoherence in the integrated ontology are the most widely proposed. However, tools that automatically detect and repair correspondences leading to changes in the original description of the input ontologies are still to be investigated and improved. Moreover, current conservativity repair systems only deal with the structural changes introduced in the integrated ontology. In addition, they generally only focus on repairing the conservativity of a single input ontology, which means that they can only resolve the asymmetric merge or enrichment case where the only repair target is the seed (priority) ontology. Overall, there is hardly any approach - among the examined ontology integration approaches - that deals with both coherence and conservativity violations at the same time, except the works of Stoilos et al. [42] and Jiménez-Ruiz et al. [55].

\subsection{Ontology Refinement}

All the research works that are mentioned in this survey did not consider refining the resulting ontology after the ontology integration process, except the work of Babalou and König-Ries [78, 99]. Therefore, another finding from this survey is that ontology refinement tools are largely missing in the ontology integration literature and still need to be addressed (See Subsection 5.2.2).

\subsection{Ontology Matching}

First, as mentioned before, it is known that most state-of-the-art ontology integration approaches are generally limited to a simple pairwise ontology matching module. In fact, throughout the last two decades, there has been a large number of approaches dealing with pairwise ontology matching. They have increased not only in number but also in performance. This is due to the availability of many benchmarks for evaluating simple pairwise ontology matching approaches. 
Second, in open, distributed and heterogeneous systems such as the Semantic Web, matching and integrating networks of ontologies (i.e., the holistic ontology matching and integration) may be very beneficial to further improve interoperability and scalability. However, most of the current ontology integration approaches are limited to matching and integrating only a pair of ontologies, while only a few systems manage multiple ontologies at once. This is due to the pairwise ontology matching strategy that gained much more attention than did the holistic ontology matching one. Holistic ontology matching is rarely considered in the literature because of its complexity and the lack of benchmarks for evaluating holistic matching approaches. As a result, current alignment repair systems also do not deal with the integration of networks of ontologies. As discussed in Section 8, they are still dedicated to only repairing an alignment between two ontologies. Future works on the ontology alignment repair area can be oriented towards this direction.

Third, most research works in the area of ontology matching remain focused on the identification of simple equivalence correspondences between ontological entities, which is the simplest ontology matching case. The performance of this task is becoming more and more efficient. Only a few systems try to discover more complex correspondences or compute different relations other than equivalence, such as subsumption, disjointness or named relations. In recent years, there has been a growing interest in the complex matching because it is one of the future directions of the ontology matching area that aims to support more elaborated and sophisticated approaches. Therefore, the integration of several ontologies with complex correspondences is a big challenge, as neither complex matching nor holistic matching is well addressed in the ontology matching area.

Further, most ontology integration approaches focus on dealing with a particular domain and cannot work with input ontologies that belong to a domain other than the one that has been predefined. This is due to their underlying ontology matching approach that does not apply to diverse domains. Only a few ontology matching tools aim at being generic and therefore suitable for several input domains or any input domain.

Finally, ontologies produced on the Web can be incrementally large. It is necessary to rely on scalable techniques in the ontology integration process. The problem of integrating large ontologies still needs to be better addressed because only a few ontology matching systems are capable of adequately handling violations in such a large matching space, e.g., the ServOMap tool [125]. Along the same line, scalability issues are only rarely considered and addressed by state-of-the-art ontology integration works.

\subsection{Ontology Integration Evaluation}

Despite the endeavor of the OAEI initiative in the creation of ontology matching benchmarks, the ontology integration area is still considerably lacking benchmarks. Ontology integration approaches often perform different integration types, use many parameters as input, make experiments on different input ontologies (two or several ontologies), and apply various evaluation metrics. As a consequence, integration results will considerably differ (depending on the chosen ontologies, integration type, parameters, and metrics). This situation makes a fair comparison between different integration tools even harder. To make future works on ontology integration comparable, it would be compelling to have a common selection of benchmarks. Such benchmarks would serve as a grand basis for evaluating ontology integration approaches in both the qualitative and quantitative performances. The creation of benchmarks can be a thriving factor in the promotion of the ontology integration area. Unlike the ontology integration area, this has been done for a long time in the ontology matching area, notably thanks to the annual OAEI campaign that has been carried out since 2004.

We conclude that the ontology integration problem depends on the performance of the ontology matching module and the quality of its generated alignments, i.e., the quality of the alignment resulting from the ontology matching process will have a direct influence on the quality of the integrated output ontology. We believe that the future of the ontology integration area is closely dependent on the future of the ontology matching area. In addition, it is clear that the creation of benchmarks can drive innovation in both the ontology integration area and the ontology matching area.

\section{Conclusion}

This survey stands at the crossroads of the domains of ontology matching and ontology integration. We reported consensual definitions of ontology integration and ontology merging, which are two close and similar notions, and we identified three types of ontology integration, namely the simple merge, the full merge, and the asymmetric merge (or the ontology enrichment). This survey has revealed that the unique type of integration that can satisfy all ontology 
integration principles is the ontology enrichment case. We also described the general principles that should be ideally fulfilled by ontology integration approaches, and reported different strategies to repair the potential issues that can arise in an output integrated ontology. Next, we outlined the most relevant ontology integration research works, starting from the pioneering works to the most recent ones. Moreover, we have shown that there is a large number of research works that integrate different input ontologies and use different evaluation measures to assess the quality of their resulting integrated ontology. In an attempt to standardize the evaluation methods, we gathered all evaluation measures from different works. The investigation on ontology integration benchmarks has just started and might receive increasing attention in the near future. Last but not least, we explored the case of integrating a network of ontologies (i.e., the case of integrating multiple ontologies using pairwise alignments) in order to investigate its particular issues and challenges. We believe that there are promising research directions in repairing conservativity violations in general, repairing coherence violations in networks of ontologies, creating recognized benchmarks-at least for two small ontologies, for any integration type - and dealing with large ontologies and scalability issues. We hope this brief exploration can provide new insights into future works in the areas of ontology matching and integration.

\section{References}

[1] J. De Bruijn, M. Ehrig, C. Feier, F. Martín-Recuerda, F. Scharffe, M. Weiten, Ontology mediation, merging and aligning, Semantic Web Technologies (2006) 95-113. doi : 10.1002/047003033X.ch6

[2] L. Predoiu, C. Feier, F. Scharffe, J. de Bruijn, F. Martín-Recuerda, D. Manov, M. Ehrig, State-of-the-art survey on ontology merging and aligning V2, EU-IST Integrated Project IST-2003-506826 SEKT (2005) 79.

[3] G. Diallo, Efficient building of local repository of distributed ontologies, in: 2011 Seventh International Conference on Signal Image Technology Internet-Based Systems, 2011, pp. 159-166. doi:10.1109/SITIS.2011.45

[4] H. S. Pinto, A. Gómez-Pérez, J. P. Martins, Some issues on ontology integration, in: the IJCAI-99 Workshop on Ontologies and ProblemSolving Methods, Vol. 18, CEUR Workshop Proceedings, 1999, pp. 7-1-7-12.

[5] H. S. Pinto, Towards Ontology Reuse, in: Proceedings of AAAI99's Workshop on Ontology Management, WS-99, Vol. 13, 1999, pp. 67-73.

[6] H. S. Pinto, J. P. Martins, Ontologies: How can they be built?, Knowledge and information systems 6 (4) (2004) $441-464$.

[7] P. Lambrix, H. Tan, SAMBO - A system for aligning and merging biomedical ontologies, Journal on Web Semantics 4 (3) (2006) $196-206$. doi:10.1016/j.websem.2006.05.003

[8] S. K. Kumar, J. A. Harding, Description logic-based knowledge merging for concrete-and fuzzy-domain ontologies, Journal of Engineering Manufacture 230 (5) (2016) 954-971. doi:10.1177/0954405414564404

[9] K. Dramé, G. Diallo, F. Delva, J. F. Dartigues, E. Mouillet, R. Salamon, F. Mougin, Reuse of termino-ontological resources and text corpora for building a multilingual domain ontology: An application to alzheimer's disease, Journal of Biomedical Informatics 48 (2014) 171 - 182. doi:https://doi.org/10.1016/j.jbi.2013.12.013

[10] P. A. Bernstein, J. Madhavan, E. Rahm, Generic schema matching, ten years later, PVLDB 4 (11) (2011) 695-701.

[11] Y. Kalfoglou, W. M. Schorlemmer, Ontology Mapping: The state of the art, The knowledge engineering review 18 (1) (2003) 1-31. doi:10.1017/S0269888903000651

[12] N. Choi, I. Song, H. Han, A survey on ontology mapping, ACM SIGMOD Record 35 (3) (2006) 34-41. doi:10.1145/1168092.1168097

Euzenat, P. Shvaiko, Ontology Matching, Springer, 2007 doi:10.1007/978-3-540-49612-0

[14] M. Granitzer, V. Sabol, K. W. Onn, D. Lukose, K. Tochtermann, Ontology alignment - A survey with focus on visually supported semiautomatic techniques, Future Internet 2 (3) (2010) 238-258. doi:10.3390/fi2030238

[15] E. Rahm, Towards large-scale schema and ontology matching, in: Schema Matching and Mapping, Data-Centric Systems and Applications, Springer, 2011, pp. 3-27. doi:10.1007/978-3-642-16518-4\_1

[16] P. Shvaiko, J. Euzenat, Ontology matching: State of the art and future challenges, IEEE Transactions on knowledge and Data Engineering 25 (1) (2013) 158-176. doi:10.1109/TKDE. 2011.253

[17] J. Euzenat, P. Shvaiko, Ontology Matching, Second Ed., Springer, 2013.

[18] L. Otero-Cerdeira, F. J. Rodríguez-Martínez, A. Gómez-Rodríguez, Ontology matching: A literature review, Expert Systems with Applications 42 (2) (2015) 949-971. doi:10.1016/j.eswa.2014.08.032

[19] P. Ochieng, S. Kyanda, Large-scale ontology matching: State-of-the-art analysis, ACM Computing Surveys 51 (4) (2018) 75:1-75:35. doi:10.1145/3211871

[20] R. Studer, V. R. Benjamins, D. Fensel, et al., Knowledge Engineering: Principles and Methods, Data and knowledge engineering 25 (1) (1998) 161-198. doi:10.1016/S0169-023X(97)00056-6

[21] O. Lassila, R. R. Swick, W. Wide, W. Consortium, Resource description framework (RDF) model and syntax specification, citeseer (1998).

[22] E. Miller, An introduction to the resource description framework, Bulletin of the American Society for Information Science and Technology 25 (1) (1998) 15-19. doi:10.1002/bult. 105

[23] D. Brickley, R. V. Guha, A. Layman, Resource description framework (RDF) schema specification, technical report, W3C, 1999. W3C Proposed Recommendation. (1999).

[24] S. Bechhofer, F. Van Harmelen, J. Hendler, I. Horrocks, D. L. McGuinness, P. F. Patel-Schneider, L. A. Stein, et al., OWL web ontology language reference W3C recommendation (February 2004).

URL https://www.w3.org/TR/owl-ref/

[25] B. Motik, P. F. Patel-Schneider, B. Parsia, C. Bock, A. Fokoue, P. Haase, R. Hoekstra, I. Horrocks, A. Ruttenberg, U. Sattler, et al., OWL 2 
web ontology language: Structural specification and functional-style syntax W3C recommendation (December 2012). URL https://www.w3.org/TR/owl2-syntax/

10.1007/978-3-319-49340-4\_8

[33] É. Thiéblin, O. Haemmerlé, N. Hernandez, C. T. dos Santos, Towards a complex alignment evaluation dataset., in: OM@ ISWC, 2017, pp. 217-218.

[34] E. Thiéblin, O. Haemmerlé, C. Trojahn, Complex matching based on competency questions for alignment: A first sketch, in: Proc. of the International Workshop on Ontology Matching co-located with the International Semantic Web Conference, OM@ISWC, Vol. 2288, 2018, pp. 66-70.

[35] E. Rahm, The case for holistic data integration, in: Proc. of the East European Conference on Advances in Databases and Information Systems, Springer, 2016, pp. 11-27. doi:10.1007/978-3-319-44039-2\_2

[36] I. Megdiche, O. Teste, C. Trojahn, An extensible linear approach for holistic ontology matching, in: the International Semantic Web Conference (ISWC), Springer, 2016, pp. 393-410. doi:10.1007/978-3-319-46523-4\_24

[37] T. Gruetze, C. Böhm, F. Naumann, Holistic and scalable ontology alignment for linked open data., WWW2012 Workshop on Linked Data on the Web (LDOW) 937 (2012).

[38] C. Pesquita, M. Cheatham, D. Faria, J. Barros, E. Santos, F. M. Couto, Building reference alignments for compound matching of multiple ontologies using obo cross-products, in: the 9th International Workshop on Ontology Matching collocated with the 13th ISWC, 2014, pp. $172-173$.

[39] D. P. d. S. Oliveira, Compound matching of biomedical ontologies, Ph.D. thesis, Universidade De Lisboa, Faculdade De Ciencias (2015).

[40] J. David, J. Euzenat, F. Scharffe, C. T. dos Santos, The alignment API 4.0, Semantic Web 2 (1) (2011) 3-10. doi:10.3233/SW-2011-0028

[41] C. Meilicke, H. Stuckenschmidt, A. Tamilin, Reasoning support for mapping revision, Journal of logic and computation 19 (5) (2008) 807-829. doi:10.1093/logcom/exn047

[42] G. Stoilos, D. Geleta, J. Shamdasani, M. Khodadadi, A novel approach and practical algorithms for ontology integration, in: Proc. of ISWC, Springer, 2018, pp. 458-476. doi:10.1007/978-3-030-00671-6\_27

[43] D. Faria, C. Pesquita, E. Santos, M. Palmonari, I. F. Cruz, F. M. Couto, The AgreementMakerLight ontology matching system, in: Proc. of the OTM 2013 Conferences - Confederated International Conferences, Springer, 2013, pp. 527-541. doi : 10.1007/978-3-642-41030-7\_38

[44] A. Solimando, E. Jimenez-Ruiz, G. Guerrini, Minimizing conservativity violations in ontology alignments: Algorithms and evaluation, Knowledge and Information Systems 51 (3) (2017) 775-819. doi:10.1007/s10115-016-0983-3

[45] A. Zimmermann, C. Le Duc, Reasoning with a network of aligned ontologies, in: the International Conference on Web Reasoning and Rule Systems, Springer, 2008, pp. 43-57. doi:10.1007/978-3-540-88737-9\_5

[46] C. Le Duc, M. Lamolle, A. Zimmermann, An API for distributed reasoning on networked ontologies with alignments., in: Proceedings of the International Conference on Knowledge Engineering and Ontology Development, (KEOD), 2010, pp. $295-304$.

[47] L. Serafini, A. Borgida, A. Tamilin, Aspects of distributed and modular ontology reasoning, in: Proc. of IJCAI, Vol. 5, 2005 , pp. 570-575.

[48] P. Hitzler, M. Krötzsch, M. Ehrig, Y. Sure, What is ontology merging?, in: American Association for Artificial Intelligence, AAAI Press, 2005, p. 4

[49] W. Lou, R. Pi, H. Wang, Y. Ju, Low-cost similarity calculation on ontology fusion in knowledge bases, Journal of Information Science. (2019) 14doi:10.1177/0165551519870456

[50] P. Mitra, G. Wiederhold, M. Kersten, A graph-oriented model for articulation of ontology interdependencies, in: Proc. of the International Conference on Extending Database Technology, Springer, 2000, pp. 86-100. doi : 10.1007/3-540-46439-5\_6

[51] C. Meilicke, H. Stuckenschmidt, An efficient method for computing alignment diagnoses, in: International Conference on Web Reasoning and Rule Systems, 2009, pp. 182-196. doi:10.1007/978-3-642-05082-4\_13

[52] J. Kim, M. Jang, Y.-G. Ha, J.-C. Sohn, S. J. Lee, MoA: OWL ontology merging and alignment tool for the semantic web, in: the International Conference on Industrial, Engineering and Other Applications of Applied Intelligent Systems, 2005, pp. 722-731. doi: $10.1007 / 11504894 \backslash \_100$

[53] J. Heflin, J. Hendler, Dynamic Ontologies on the Web, in: Proc. of AAAI/IAAI, AAAI Press / The MIT Press, 2000, pp. 443-449.

[54] M. Uschold, Achieving semantic interoperability using RDF and OWL-v10, 2005 Last accessed 13 Nov 2020 (2005). URL https://www.w3.org/2001/sw/BestPractices/OEP/SemInt/

[55] E. Jiménez-Ruiz, B. C. Grau, I. Horrocks, R. B. Llavori, Ontology Integration Using Mappings: Towards Getting the Right Logical Consequences, in: Proc. of the 6th European Semantic Web Conference (ESWC), Springer, 2009, pp. 173-187. doi:10.1007/ 978-3-642-02121-3\_16

[56] P. Bouquet, F. Giunchiglia, F. Van Harmelen, L. Serafini, H. Stuckenschmidt, C-OWL: Contextualizing ontologies, in: Proc. of the International Semantic Web Conference (ISWC), Springer, 2003, pp. 164-179. doi:10.1007/978-3-540-39718-2\_11

[57] A. Borgida, L. Serafini, Distributed description logics: Assimilating information from peer sources, Journal on data semantics I 1 (2003) 153-184. doi:10.1007/978-3-540-39733-5\_7 
[58] B. C. Grau, B. Parsia, E. Sirin, Working with multiple ontologies on the semantic web, in: the International Semantic Web Conference (ISWC), Springer, 2004, pp. 620-634. doi:10.1007/978-3-540-30475-3\_43

[59] I. Horrocks, P. F. Patel-Schneider, H. Boley, S. Tabet, B. Grosof, M. Dean, et al., SWRL: A semantic web rule language combining OWL and RuleML, W3C Member submission 21 (79) (2004) 1-31.

[60] A. Miles, S. Bechhofer, SKOS simple knowledge organization system reference, W3C recommendation 18 (2009) W3C.

[61] C. Meilicke, Alignment Incoherence in Ontology Matching, Ph.D. thesis, Universität Mannheim (2011).

[62] J. Sowa, Electronic communication in the onto-std mailing list (December 1997).

[63] S. Raunich, E. Rahm, Atom: Automatic target-driven ontology merging, in: Proc. of the 27th International Conference on Data Engineering, ICDE 2011, IEEE, 2011, pp. 1276-1279. doi:10.1109/ICDE. 2011.5767871

[64] S. Raunich, E. Rahm, Target-driven Merging of Taxonomies with ATOM, Information Systems 42 (2014) 1-14. doi:10.1016/j . is .2013 11.001

[65] R. Pottinger, P. A. Bernstein, Merging models based on given correspondences, in: Proceedings of the 29th International Conference on Very Large Data Bases (VLDB 2003), Morgan Kaufmann, 2003, pp. 826-873. doi:10.1016/B978-012722442-8/50081-1

[66] S. Babalou, B. König-Ries, GMRs: Reconciliation of generic merge requirements in ontology integration in: Proc. of the Posters\&Demos Track of the International Conference on Semantic Systems (SEMANTiCS), Vol. 2451, 2019, p. 5. URL http://ceur-ws.org/Vol-2451/paper-04.pdf

[67] U. Sattler, R. Stevens, P. Lord, (I can’t get no) satisfiability] Ontogenesis, Last accessed 13 Nov 2020 (2013) URL http://ontogenesis.knowledgeblog.org/1329

[68] S. Bail, Common reasons for ontology inconsistency. Ontogenesis, Last accessed 13 Nov 2020 (2013). URL http://ontogenesis.knowledgeblog.org/1343

[69] M. Fahad, N. Moalla, A. Bouras, M. A. Qadir, M. Farukh, Disjoint-Knowledge Analysis and Preservation in Ontology Merging Process, in: Proc. of the Fifth International Conference on Software Engineering Advances, ICSEA, IEEE, 2010, pp. 422-428. doi:10.1109/ICSEA 2010.72

[70] A. Solimando, E. Jiménez-Ruiz, G. Guerrini, Detecting and correcting conservativity principle violations in ontology-to-ontology mappings, in: Proc. of the International Semantic Web Conference (ISWC), Springer, 2014, pp. 1-16. doi:10.1007/978-3-319-11915-1\_1

[71] A. Solimando, E. Jiménez-Ruiz, G. Guerrini, A multi-strategy approach for detecting and correcting conservativity principle violations in ontology alignments, in: the 11th International Workshop on OWL: Experiences and Directions (OWLED) co-located with ISWC, 2014, pp. $13-24$.

[72] M. Poveda-Villalón, M. C. Suárez-Figueroa, A. Gómez-Pérez, Validating ontologies with OOPS!, in: Proceedings of the 18th International Conference on Knowledge Engineering and Knowledge Management (EKAW 2012), Vol. 7603, Springer, 2012, pp. 267-281. doi: 10.1007/978-3-642-33876-2\_24

[73] M. Poveda-Villalón, A. Gómez-Pérez, M. C. Suárez-Figueroa, OOPS! (OntOlogy Pitfall Scanner!): An on-line tool for ontology evaluation, International Journal on Semantic Web and Information Systems (IJSWIS) 10 (2) (2014) 7-34. doi:10.4018/ijswis . 2014040102

[74] F. Duchateau, Z. Bellahsene, Measuring the quality of an integrated schema, in: Proceedings of the International Conference on Conceptual Modeling, Springer, 2010, pp. 261-273. doi:10.1007/978-3-642-16373-9\_19

[75] N. F. Noy, M. A. Musen, PROMPT: Algorithm and Tool for Automated Ontology Merging and Alignment, in: Proc. of AAAI/IAAI, AAAI Press / The MIT Press, 2000, pp. 450-455.

[76] A. Guzmán-Arenas, A. Cuevas-Rasgado, Knowledge accumulation through automatic merging of ontologies, Expert Systems with Applications 37 (3) (2010) 1991-2005. doi:10.1016/j.eswa.2009.06.078

[77] J. Zhang, Y. Lv, An approach of refining the merged ontology, in: Proceedings of the 9th International Conference on Fuzzy Systems and Knowledge Discovery (FSKD 2012), IEEE, 2012, pp. 802-806. doi : 10.1109/FSKD. 2012.6233973

[78] S. Babalou, B. König-Ries, Towards building knowledge by merging multiple ontologies with CoMerger: A partitioning-based approach, CoRR abs/2005.02659 (2020). arXiv:2005.02659

[79] A. L. Rector, N. Drummond, M. Horridge, J. Rogers, H. Knublauch, R. Stevens, H. Wang, C. Wroe, OWL pizzas: Practical experience of teaching OWL-DL: common errors \& common patterns, in: Proceedings of the 14th International Conference on Knowledge Engineering and Knowledge Management (EKAW 2004), Vol. 3257, Springer, 2004, pp. 63-81. doi:10.1007/978-3-540-30202-5\_5

[80] G. Schreiber, OWL restrictions Last accessed 13 Nov 2020 (May 2005). URL https://www.cs.vu.nl/ guus/public/owl-restrictions/

[81] M. Horridge, H. Knublauch, A. Rector, R. Stevens, C. Wroe, A practical guide to building OWL ontologies using the protégé-OWL plugin and CO-ODE tools edition 1.0 University of Manchester (2004). URL https://cse.buffalo.edu/ shapiro/Courses/CSE663/Fall07/ProtegeOWLTutorial.pdf

[82] C. Meilicke, H. Stuckenschmidt, Applying logical constraints to ontology matching, in: the Annual Conference on Artificial Intelligence, Springer, 2007, pp. 99-113. doi:10.1007/978-3-540-74565-5\_10

[83] E. Jiménez-Ruiz, B. C. Grau, LogMap: Logic-based and scalable ontology matching, in: the 10th International Semantic Web Conference (ISWC), Vol. 7031, 2011, pp. 273-288. doi:10.1007/978-3-642-25073-6\_18

[84] E. Jiménez-Ruiz, B. C. Grau, Y. Zhou, I. Horrocks, Large-scale interactive ontology matching: Algorithms and implementation, in: the 20th European Conference on Artificial Intelligence ECAI, Vol. 242, IOS Press, 2012, pp. 444-449. doi:10.3233/978-1-61499-098-7-444

[85] E. Santos, D. Faria, C. Pesquita, F. M. Couto, Ontology alignment repair through modularization and confidence-based heuristics, PloS one 10 (12) (2015) e0144807. doi:10.1371/journal.pone.0144807

[86] D. Ngo, Z. Bellahsene, YAM++: A multi-strategy based approach for ontology matching task, in: Proceedings of the International Conference on Knowledge Engineering and Knowledge Management, Springer, 2012, pp. 421-425. doi:10.1007/978-3-642-33876-2\_38

[87] Y. R. Jean-Mary, E. P. Shironoshita, M. R. Kabuka, Ontology matching with semantic verification, Journal of Web Semantics 7 (3) (2009) 235-251. doi:10.1016/j.websem.2009.04.001

[88] P. Wang, Y. Zhou, B. Xu, Matching large ontologies based on reduction anchors, in: Proc. of the Twenty-Second International Joint Conference on Artificial Intelligence, (IJCAI), 2011, pp. 2343-2348. doi:10.5591/978-1-57735-516-8/IJCAI11-390 
[89] S. Schlobach, R. Cornet, et al., Non-standard reasoning services for the debugging of description logic terminologies, in: Proc. of IJCAI, Vol. 3, 2003, pp. 355-362.

[107] E. G. Caldarola, A. M. Rinaldi, An approach to ontology integration for ontology reuse, in: the International Conference on Information Reuse and Integration, IRI, IEEE, 2016, pp. 384-393. doi:10.1109/IRI.2016.58

[108] N. F. Noy, M. A. Musen, The PROMPT suite: interactive tools for ontology merging and mapping, International Journal of Human-Computer Studies 59 (6) (2003) 983-1024. doi:10.1016/j.ijhcs.2003.08.002

[109] D. L. McGuinness, R. Fikes, J. Rice, S. Wilder, The Chimaera Ontology Environment, in: Proc. of AAAI/IAAI, Vol. 2000, AAAI Press / The MIT Press, 2000, pp. 1123-1124.

[110] G. Stumme, A. Maedche, FCA-MERGE: Bottom-Up merging of ontologies, in: Proceedings of the Seventeenth International Joint Conference on Artificial Intelligence (IJCAI), Morgan Kaufmann, 2001, pp. 225-234.

[111] D. Dou, D. V. McDermott, P. Qi, Ontology Translation on the Semantic Web, in: Proc. of the OTM Confederated International Conferences, CoopIS, DOA, and ODBASE: On the Move to Meaningful Internet Systems, Springer, 2003, pp. 952-969. doi: 10.1007/978-3-540-39964-3\_60

[112] K. Kotis, G. A. Vouros, K. Stergiou, Towards automatic merging of domain ontologies: The HCONE-merge approach, Journal on Web semantics 4 (1) (2006) 60-79. doi:10.1016/j.websem.2005.09.004

[113] O. Udrea, L. Getoor, R. J. Miller, Leveraging Data and Structure in Ontology Integration, in: the SIGMOD International Conference on Management of Data, ACM, 2007, pp. 449-460. doi:10.1145/1247480.1247531

[114] M. Maree, M. Belkhatir, A coupled statistical/semantic framework for merging heterogeneous domain-specific ontologies, in: Proc. of the 22nd IEEE International Conference on Tools with Artificial Intelligence, (ICTAI 2010), Vol. 2, IEEE, 2010, pp. 159-166. doi: 10.1109/ICTAI.2010.138

[115] M. Maree, M. Belkhatir, Addressing semantic heterogeneity through multiple knowledge base assisted merging of domain-specific ontologies, Knowledge-Based Systems 73 (2015) 199-211. doi:10.1016/j.knosys.2014.10.001

[116] M. Fahad, N. Moalla, A. Bouras, Detection and resolution of semantic inconsistency and redundancy in an automatic ontology merging system, Journal of Intelligent Information Systems. 39 (2) (2012) 535-557. doi:10.1007/s10844-012-0202-y

[117] L. Zhao, R. Ichise, Ontology Integration for Linked Data, Journal on Data Semantics 3 (4) (2014) 237-254. doi:10.1007/ s13740-014-0041-9

[118] Z. El Jerroudi, J. Ziegler, iMERGE: Interactive ontology merging, in: Proceedings of the International Conference on Knowledge Engineering and Knowledge Management (EKAW 2008), Springer, 2008, pp. 52-56.

[119] M. Mahfoudh, G. Forestier, M. Hassenforder, A Benchmark for Ontologies Merging Assessment, in: Proc. of the International Conference on 
Knowledge Science, Engineering and Management (KSEM), Springer, 2016, pp. 555-566. doi:10.1007/978-3-319-47650-6\_44

1120] M. Horridge, S. Bechhofer, The OWL API: A Java API for OWL ontologies, Semantic Web 2 (1) (2011) 11-21. doi:10.3233/ SW-2011-0025

[121] O. Bodenreider, The Unified Medical Language System (UMLS): Integrating biomedical terminology, Nucleic acids research 32 (DatabaseIssue) (2004) 267-270. doi:10.1093/nar/gkh061

[122] Y. Kazakov, M. Krötzsch, F. Simancik, ELK Reasoner: Architecture and Evaluation., in: Proceedings of the 1st International Workshop on OWL Reasoner Evaluation (ORE), Vol. 858, CEUR-WS.org, 2012, p. 12.

[123] C. Pesquita, D. Faria, E. Santos, F. M. Couto, To repair or not to repair: Reconciling correctness and coherence in ontology reference alignments, in: the Ontology Matching Workshop (OM) co-located with the International Semantic Web Conference (ISWC), CEUR-WS.org, 2013, pp. 13-24.

[124] E. Jiménez-Ruiz, B. C. Grau, I. Horrocks, R. Berlanga, Logic-based assessment of the compatibility of UMLS ontology sources, Journal of Biomedical Semantics 2 (1) (2011) S2.

[125] G. Diallo, An effective method of large scale ontology matching, Journal of Biomedical Semantics 5 (1) (2014) 44. doi:10.1186/ 2041-1480-5-44 